\title{
1 Non-invasive human skin transcriptome analysis using mRNA in skin
}

\section{2 surface lipids}

4 Takayoshi Inoue*, Tetsuya Kuwano, Yuya Uehara, Michiko Yano, Naoki Oya, Akira

5 Hachiya, Yoshito Takahashi, Noriyasu Ota, Takatoshi Murase*

6

7 Biological Science Research, Kao Corporation, Tochigi, Japan

8 2606, Akabane, Ichikai-machi, Haga-gun, Tochigi, 321-3497, Japan

\section{*Corresponding authors}

11 Takayoshi Inoue, PhD; E-mail: inoue.takayoshi@kao.com 


\section{$17 \underline{\text { Abstract }}$}

18 Non-invasive acquisition of mRNA data from the skin would be extremely useful for

19 understanding skin physiology and diseases. Inspired by the holocrine process, in which the

20 sebaceous glands secrete cell contents into the sebum, we focused on the possible presence of

21 mRNAs in skin surface lipids (SSLs). We found that measurable human mRNAs exist in SSLs,

22 where sebum protects them from degradation by RNases. The AmpliSeq transcriptome analysis

23 was modified to measure SSL-RNAs, and our results revealed that SSL-RNAs predominantly

24 contained mRNAs derived from sebaceous glands, epidermis, and hair follicles. Analysis of

25 SSL-RNAs non-invasively collected from patients with atopic dermatitis revealed significantly

26 increased expression of inflammation-related genes and decreased expression of terminal

27 differentiation-related genes, consistent with the results of previous reports. Further, we found that lipid synthesis-related genes were downregulated in the sebaceous glands of patients with atopic dermatitis. These results indicate that the analysis of SSL-RNAs is promising to understand the pathophysiology of skin diseases. 


\section{$\underline{\text { Introduction }}$}

Intra- and inter-organ communication mediated via various hormones, growth factors,

cytokines, metabolites, and miRNAs play important roles in maintaining homeostasis in the

for these mediators to monitor the physiological conditions of the body and explore predictive

biomarkers for various diseases (2-5). Especially, the use of serum, urine, and saliva samples,

which can be obtained in a non- or low-invasive manner, has been widely investigated.

as the cutaneous pathology, appearance, and its secretions reflect not only the skin condition but

biomolecules can be easily collected from the sweat, hair, and stratum corneum samples, and

thus, skin is a useful source of samples to monitor the skin and body conditions. For instance,

the cortisol content in the scalp hair correlates with long-term cumulative cortisol exposure (7).

The sweat can also be used as an indicator of internal physiological changes (8), and attempts

have been made to monitor patients' conditions, for instance, tracking blood glucose levels by

measuring glucose in sweat samples of patients with diabetes (9). Although metabolites, 
50 stripped stratum corneum has been used to collect mRNAs in a minimally invasive manner (12),

51 however, the mRNA content is very low and highly degraded due to the RNase activity on the

52 skin surface. Therefore, a skin biopsy is practically required to analyze mRNA expression;

53 however, this method is invasive, which limits its application. More recently, a minimally

54 invasive method for mRNA analysis via RNA-seq using AmpliSeq technology with 16-20

consecutive tape strips was reported (13-15). However, tape stripping of the stratum corneum is

known to induce skin damage, including disruption of the skin barrier (16), epidermal

hyperproliferation, and infiltration of CD3-positive $\mathrm{T}$ cells into the dermis (17), indicating that

the problems related to the invasiveness of this technique remain to be fully resolved.

59 The sebaceous glands synthesize and accumulate lipids to produce sebum. The lipids

60 accumulated in the cytosol of sebocytes are secreted into the sebaceous ducts following rupture

61 of the plasma membrane; this mode of secretion is called holocrine secretion (18) and is unique

among the exocrine glands such as lipid-secreting sebaceous and meibomian glands. The

holocrine secretion of the cell contents led us to the idea that sebum may contain various

biomolecules, including mRNAs, which may be useful for analyzing biological information.

Therefore, in this study, we first investigated the presence of mRNAs in human sebum and 
bioRxiv preprint doi: https://doi.org/10.1101/2021.04.04.438351; this version posted April 5, 2021. The copyright holder for this preprint (which was not certified by peer review) is the author/funder, who has granted bioRxiv a license to display the preprint in perpetuity. It is made available under aCC-BY-NC-ND 4.0 International license.

68 characterization was verified in healthy subjects and patients with atopic dermatitis (AD).

69 


\section{Subject recruitment and collection of SSLs}

Thirty-two healthy male individuals (mean age: 34.6 years, range: $20-49$ years, $S D=9.24$ )

were recruited for the study that was conducted in October 2016. The individuals were

evaluated by dermatologists prior to the commencement of the study to confirm no obvious skin

years, $\mathrm{SD}=8.82$ ) diagnosed with mild or moderate facial $\mathrm{AD}$ were recruited during June-

October 2017. All subjects were required not to remove facial sebum by washing or using wipes

or shaving their face on the test day until the end of the test. Patients with AD were also

restricted to use steroidal anti-inflammatory and immunosuppressive drugs on the facial skin 24

h prior to the study. The study was approved by the Human Research Ethics Committee, Kao 
88

89

90

92

\section{Skin tissue}

$$
\text { Surgically removed adult forehead skin from three Caucasian males, aged 62, 66, and } 67
$$

years, and nose skin from one adult (Caucasian female, 66-years-old) were provided by the

Colorado Dermatology Institute (Colorado Springs, CO, USA) for laser microdissection and

immunostaining, respectively. The procurement of skin tissues was approved by the Institutional

Review Board of IntegReview Ltd. (Austin, TX, USA; approval number: T046a-170829) and

was conducted according to the Declaration of Helsinki Principles. Informed consents were

obtained from the volunteers prior to surgery. After surgery, the skin tissues were stored in

William's E medium (Life Technologies, Carlsbad, CA, USA) at $4{ }^{\circ} \mathrm{C}$ until embedding. All skin tissues were embedded using the Tissue-Tek optimal cutting temperature compound (Sakura

Finetek, Tokyo, Japan) and kept frozen until sectioning.

\section{mRNA extraction and qPCR}

Human saliva, sweat, urine, and serum samples collected from two donors were purchased

from Cosmo Bio (Tokyo, Japan). Total RNA was extracted from $1 \mathrm{~mL}$ of each sample using the

TRIzol LS reagent (Thermo Fisher Scientific, Waltham, MA, USA). Total RNA was extracted

from the stratum corneum of the cheek of two healthy males according to a previous report (12).

Collected RNA was dissolved with $10 \mu \mathrm{L}$ of nuclease-free water. RNAs in SSLs (SSL-RNAs) 
106 was extracted using the TRIzol reagent (Thermo Fisher Scientific) as follows: $2.85 \mathrm{~mL}$ of

107 TRIzol was added to a finely cut oil blotting film containing sebum samples. Next, the solution

108 was divided equally into two tubes and $260 \mu \mathrm{L}$ of chloroform was added to each tube and mixed

109 by vortexing. The tubes were centrifuged at $12,000 \times g$ for 15 min at $4{ }^{\circ} \mathrm{C}$. The upper layer was

110 transferred to a fresh tube and precipitated with ethanol. The precipitates were washed with

$11170 \%$ ethanol (v/v) and dissolved in $10 \mu \mathrm{L}$ of nuclease-free water.

112 Reverse transcription was performed using the SuperScript IV First-Strand Synthesis

113 System and Oligo-dT primers (Thermo Fisher Scientific). The qPCR was performed using the

114 TaqMan Fast Universal PCR Master Mix (Thermo Fisher Scientific) and TaqMan probes for

115 each gene (Thermo Fisher Scientific).

Evaluation of mRNA degradation in SSLs

To prepare standard samples with different levels of degraded mRNAs, total RNA $(1 \mu \mathrm{g})$

119 extracted from normal human epidermal keratinocytes (NHEK) (Cascade Biologics, Portland,

120 OR, USA) was incubated with 30-1000 ng/mL of recombinant human RNase 7 (Novus

121 Biologicals, Littleton, CO, USA) in $10 \mathrm{mM}$ Tris-HCl buffer (pH 8.0) (Nippon Gene, Tokyo,

122 Japan) for 30 min. QIAzol lysis reagent (Qiagen, Hilden, Germany) and chloroform were added

123 to the samples treated with RNase 7 or SSLs collected from six healthy males as described 
124 above. After the solutions were vortexed and centrifuged, RNA in the supernatant was purified

125 using the miRNeasy Mini Kit (Qiagen). The level of RNA degradation in the samples was

126 determined using the High Sensitivity RNA ScreenTape (Agilent Technologies, Palo Alto, CA,

127 USA) on an Agilent 4200 TapeStation system (Agilent Technologies). The level of degradation

128 of human mRNAs in SSLs was estimated using the following calculated DV200 value, which

129 evaluates the percentage of fragments containing $>200$ nucleotides. mRNA was reverse-

130 transcribed using the SuperScript IV First-Strand Synthesis System and Oligo-dT primers

131 (Thermo Fisher Scientific) and was amplified in a thermal cycler using the PowerUP SYBR

132 Green Master Mix (Thermo Fisher Scientific) and the following primers:

133 ACTB (forward primer $57 \mathrm{bp}$ ): 5'-GCTTTTGGTCTCCCTGGGAG-3'

$134 A C T B$ (forward primer 363 bp): 5'-ACAATGTGGCCGAGGACTTT-3'

135 ACTB (reverse primer): 5'-AGTCAGTGTACAGGTAAGCCC-3'

136 Abundance of each amplicon was determined from the RNA standard curve. Further,

137 DV200 values of the SSL-RNA samples were calculated from the standard curve of DV200

138 plotted against abundance ratio of the amplicons (363 bp/57 bp).

\section{Immunostaining}

141 Skin sections obtained from the nose of a Caucasian female were fixed in acetone at $-20{ }^{\circ} \mathrm{C}$ 
142 for $10 \mathrm{~min}$ and treated with $0.1 \%$ Triton X-100/PBS for 5 min. The skin sections were

143 incubated with Protein Block serum-free (Agilent Technologies) for $30 \mathrm{~min}$, then with the

144 primary antibodies against keratin/cytokeratin (mouse mono-clonal (AE-1/AE-3), original

145 solution, Nichirei, Tokyo, Japan) or RNase 7 (rabbit poly-clonal, 1:50, Cloud-Clone Corp.,

146 Houston, TX, USA) for $1 \mathrm{~h}$ at $20-25^{\circ} \mathrm{C}$, and finally with anti-rabbit IgG (donkey poly-clonal,

147 Alexa Fluor 555, 1:1000, Thermo Fisher Scientific) or anti-mouse IgG (goat poly-clonal, Alexa

148 Fluor 647, 1:1000, Thermo Fisher Scientific) for $30 \mathrm{~min}$. Samples were mounted on a glass slide

149 and imaged using fluorescence microscopy (BZ-X710, Keyence, Osaka, Japan).

Western blotting

Total protein was extracted from SSLs using $350 \mu \mathrm{L}$ of RIPA buffer and was purified using

the Ready Prep 2-D cleanup kit (Bio-Rad, Hercules, CA, USA); the protein concentration was

measured using the BCA protein assay kit (Thermo Fisher Scientific). Afterwards, $70 \mu \mathrm{L}$ of

trichloroacetic acid was added and the samples were incubated on ice for $30 \mathrm{~min}$, followed by

centrifugation at $13,000 \times g$ for $5 \mathrm{~min}$ at $4{ }^{\circ} \mathrm{C}$. Chilled acetone $(500 \mu \mathrm{L})$ was added to the

pellets, the tubes were centrifuged at $13,000 \times g$ for 5 min at $4{ }^{\circ} \mathrm{C}$, and the supernatant was 
160 gel (Bio-Rad) and then transferred to a polyvinylidene fluoride (PVDF) membrane (Bio-Rad)

161 soaked in Tris-Glycine Buffer (25 mM Tris, $192 \mathrm{mM}$ glycine; $\mathrm{pH}$ 8.2) containing $20 \%$ (v/v)

162 methanol. The PVDF membrane blocked with PVDF Blocking Reagent for Can Get Signal

163 (Toyobo, Tokyo, Japan) was incubated with anti-RNase 7 antibody (rabbit polyclonal, 1:200,

164 Cloud-Clone Corp, Katy, TX, USA) for 60 min followed by incubation with anti-rabbit IgG,

165 horseradish peroxidase-linked secondary antibody (donkey monoclonal, 1:2000, GE Healthcare,

166 Bucks, UK) for $45 \mathrm{~min}$. The bands were visualized using ECL prime western blotting detection

reagents (GE Healthcare).

Effect of sebum lipids on RNase activity

water and $4 \mathrm{~mL}$ tert-butyl methyl ether were added to the cut film. The solution was transferred

transferred to a new glass vial, and the organic solvent was dried by blowing nitrogen over it.

The remaining sebum lipids were dissolved in $100 \mu \mathrm{L}$ dimethyl sulfoxide (DMSO) and used for analysis. 
178 triacylglycerol (glyceryl trioleate, Sigma-Aldrich, T7140), free fatty acid (palmitoleic acid,

179 Sigma-Aldrich, P9417), squalene (Sigma-Aldrich, S3626), and cholesterol (Sigma-Aldrich,

180 C8667) were used as authentic samples to identify the lipid molecular species. The sebum lipids

181 extracted from the oil blotting films were dissolved in chloroform $/$ methanol $(2: 1, \mathrm{v} / \mathrm{v})$. The

182 authentic lipids and $5 \mathrm{mg}$ sebum lipid samples were separated on a thin-layer chromatography

183 (TLC) plate using hexane: diethyl ether: acetic acid (70:30:1, v/v/v). After chromatography, the

184 plate was divided using a glass cutter into portions containing the authentic lipids and sebum

185 lipid samples, and the portion containing the authentic lipids was sprayed with a solution

186 containing $10 \%(\mathrm{w} / \mathrm{v})$ copper sulfate and $8 \%(\mathrm{w} / \mathrm{v})$ phosphoric acid, and then heated at $180{ }^{\circ} \mathrm{C}$

187 for 3 min to visualize the location of each lipid. Based on the mobility of the authentic lipids,

188 the silica corresponding to portions of sebum lipids was scraped off. The silica was sonicated in

189 the chloroform/methanol $(2: 1, \mathrm{v} / \mathrm{v})$ mixture to extract the lipids and centrifuged at $2,050 \times \mathrm{g}$ for

1905 min. The supernatant was dried and dissolved in $15 \mu \mathrm{L}$ DMSO; the solution was further

191 diluted 3- and 9-fold using DMSO.

192 Cholesterol ester (cholesteryl palmitoleate, Olbracht Serdary Research Laboratories,

193 Toronto, Canada, D-161), wax ester (behenyl palmitoleate, Nu Chek Prep, Elysian, MN, USA,

194 WE-1368), triacylglycerol (glyceryl tripalmitoleate, Sigma-Aldrich, T5888; glyceryl trioleate,

195 Sigma-Aldrich, T7140), free fatty acids (myristoleic acid, Sigma-Aldrich, M3525; palmitoleic 
196 acid, Sigma-Aldrich, P9417; oleic acid, Sigma-Aldrich, O1008), squalene (Sigma-Aldrich,

197 S3626), and cholesterol (Sigma-Aldrich, C8667) were used to determine the active ingredients

198 in the sebum. NHEK RNA ( $20 \mu \mathrm{g} / \mathrm{mL}$ final concentration) was mixed with RNase 7 (final

199 concentration: $1 \mu \mathrm{g} / \mathrm{mL}$ ) in $10 \mathrm{mM}$ Tris- $\mathrm{HCl}$ buffer ( $\mathrm{pH} 8.0$ ) with or without sebum or the lipid

200 reagent (total $50 \mu \mathrm{L}$ ), and sonicated, followed by incubation at $20-25^{\circ} \mathrm{C}$ for $30 \mathrm{~min}$.

201 Subsequently, RNA was extracted using TRIzol LS reagent and the quality of the RNA was

202 determined using the High Sensitivity RNA ScreenTape on Agilent 4200 TapeStation System.

\section{Library preparation for Ion AmpliSeq}

206 film containing sebum samples, QIAzol solution was divided equally into two tubes.

207 Chloroform $(260 \mu \mathrm{L})$ was added to each tube and vortexed, and the tubes were centrifuged at

$12,000 \times g$ for $15 \mathrm{~min}$ at $4{ }^{\circ} \mathrm{C}$. The upper layer was transferred to a fresh tube. RNA was purified using the RNeasy mini kit (performing DNase treatment in the purification step) (Qiagen) and

precipitation, and then dissolved in $10 \mu \mathrm{L}$ of nuclease-free water. 
214 Fisher Scientific). Briefly, $1.75 \mu \mathrm{L}$ RNA solution was mixed with $0.5 \mu \mathrm{L}$ VILO Reaction Mix

215 and $0.25 \mu \mathrm{L}$ SuperScript III Enzyme. Reverse transcription was performed at $25^{\circ} \mathrm{C}$ for $10 \mathrm{~min}$,

$21642{ }^{\circ} \mathrm{C}$ for $90 \mathrm{~min}$, and finally $85^{\circ} \mathrm{C}$ for $5 \mathrm{~min}$. The target DNA amplification was performed by

217 mixing $2.5 \mu \mathrm{L}$ cDNA solution, $1.5 \mu \mathrm{L}$ nuclease-free water, $2.0 \mu \mathrm{L}$ Ion AmpliSeq HiFi Mix, and

$2184.0 \mu \mathrm{L}$ Ion AmpliSeq Transcriptome Human Gene Expression Core Panel under the following

219 conditions: $99^{\circ} \mathrm{C}$ for $15 \mathrm{sec}$ and $62^{\circ} \mathrm{C}$ for $16 \mathrm{~min}$ for 20 cycles. The amplified DNA library was

220 purified by mixing $10 \mu \mathrm{L}$ AMPure XP beads (Beckman Coulter, Miami, FL, USA) according to

221 the manufacturer's protocol and eluted using $10 \mu \mathrm{L}$ nuclease-free water. The quality check of the

222 DNA library was conducted using the High Sensitivity D1000 ScreenTape on Agilent 4200

223 TapeStation. If the DNA library had amplified, a band of approximately $170 \mathrm{bp}$ was observed.

224 After checking the DNA library quality, the reaction solution was prepared by mixing $3.5 \mu \mathrm{L}$

225 purified library solution, $2.0 \mu \mathrm{L}$ Ion AmpliSeq HiFi Mix, $4.0 \mu \mathrm{L}$ Ion AmpliSeq Transcriptome

226 Human Gene Expression Core Panel, and $0.5 \mu \mathrm{L}$ VILO Reaction Mix. After adding $1.0 \mu \mathrm{L}$ FuPa

227 reagent to $10 \mu \mathrm{L}$ reconstituted reaction solution, the primer sequence was partially digested

228 under the following conditions: $50{ }^{\circ} \mathrm{C}$ for $10 \mathrm{~min}, 55^{\circ} \mathrm{C}$ for $10 \mathrm{~min}$, and $60{ }^{\circ} \mathrm{C}$ for $20 \mathrm{~min}$. To

229 ligate the adaptor sequence, $2 \mu \mathrm{L}$ Switch solution, $1 \mu \mathrm{L}$ Ion Xpress Barcode adapters, and $1 \mu \mathrm{L}$

230 DNA ligase were added to $11 \mu \mathrm{L}$ reaction solution, followed by incubation at $22{ }^{\circ} \mathrm{C}$ for $60 \mathrm{~min}$

231 and $72{ }^{\circ} \mathrm{C}$ for $5 \mathrm{~min}$. The library $(15 \mu \mathrm{L})$ ligated with the adaptor sequence was purified via 
232 mixing with $18 \mu \mathrm{L}$ AMPure XP beads according to the manufacturer's protocol. Libraries were

233 eluted using $50 \mu \mathrm{L}$ Library Amp Mix (Thermo Fisher Scientific) to which, $2 \mu \mathrm{L}$ Library Amp

234 Primers were added. The library amplification was conducted using following steps: $98{ }^{\circ} \mathrm{C}$ for

23515 seconds and $64{ }^{\circ} \mathrm{C}$ for 1 min for five cycles. Next, $50 \mu \mathrm{L}$ PCR product was mixed with $25 \mu \mathrm{L}$

236 AMPure XP beads and the supernatant was transferred to fresh PCR tubes. The supernatants

237 were mixed with $60 \mu \mathrm{L}$ AMPure XP beads and purified; target fragments were eluted from the

238 beads using $10 \mu \mathrm{L}$ TE buffer. The quality check of the library was performed using the High

239 Sensitivity D1000 ScreenTape on Agilent 4200 TapeStation.

Sequencing

The library was quantified using the Ion Library TaqManTM Quantitation Kit (Thermo

Fisher Scientific). After an input of 50 pM DNA library in the Ion Chef System (Thermo Fisher on the Ion S5 XL System (Thermo Fisher Scientific).

Verification of the correlation coefficient between AmpliSeq and qPCR results 
250 pooled TaqMan probe ( $R P L P 0, C D S N$, and CCL17) (Thermo Fisher Scientific) and then diluted

2515 -fold with nuclease-free water. The qPCR was performed according to the aforementioned

252 "mRNA extraction and qPCR" method. Expression value of $R P L P 0$ was used as an internal

253 control. Correlation between the value of reads per million mapped reads (RPM) of AmpliSeq

254 and the relative expression value of qPCR in healthy subjects and patients with AD was

255 analyzed.

\section{Laser microdissection (LMD) and AmpliSeq transcriptome analysis}

Frozen skin sections (thickness: $10 \mu \mathrm{m}$ ) from three Caucasian males were mounted on

membrane slides (PEN-Membrane $2.0 \mu \mathrm{m}$, Leica Microsystems, Wetzlar, Germany) treated

with $0.1 \%$ (w/v) poly-L-lysine (Fujifilm Wako Pure Chemical, Osaka, Japan). In addition, two

frozen sections were directly collected into $750 \mu \mathrm{L}$ RLT buffer (Qiagen) containing $40 \mathrm{mM}$

sections were fixed with acetone at $-20{ }^{\circ} \mathrm{C}$ for $10 \mathrm{~min}$, stained with $0.05 \%(\mathrm{v} / \mathrm{v})$ toluidine blue,

and finally dried. Epidermis, sebaceous glands, sweat glands, hair follicles, and dermis were

carefully microdissected from the skin sections using LMD7000 (Leica Microsystems). Fifteen 
268 kit (performing DNase treatment in the purification step) (Qiagen). The RNA was concentrated

269 via ethanol precipitation and dissolved in $10 \mu \mathrm{L}$ nuclease-free water. The RNA quality was

270 checked on the 4200 TapeStation System and cDNA library was prepared for AmpliSeq

271 transcriptome sequencing using 75 pg total RNA according to the method described in "Library

272 preparation for Ion AmpliSeq". The number of target amplification was changed from 20 to 18

273 cycles except for dermis samples.

Statistics, normalization, and differential expression analysis of the AmpliSeq whole

transcriptome

using the R statistical language. Read counts were generated using the AmpliSeq RNA plugin in

patients with $\mathrm{AD}$, a likelihood ratio test was performed with DESeq2 using normalized counts.

Heat maps were generated using the heatmap3 package. Dimensionality reduction using t- 


\section{$286 \quad$ Results}

\section{Measurable human mRNA is present in SSLs}

qPCR was conducted to evaluate the mRNA abundance in SSL samples, stratum corneum,

289 urine, serum, saliva, and sweat samples. The expression of $A C T B$ and GAPDH mRNA in SSLs

290 was comparable to their expression in $100 \mathrm{ng}-500 \mathrm{pg}$ and $1 \mathrm{ng}-100 \mathrm{ng}$ total RNA in NHEK,

291 respectively, whereas these transcripts were largely undetectable in the other body fluid samples

292 (Fig. 1a). The level of human mRNA degradation in SSLs could not be measured directly due to

293 the presence of bacterial mRNA. Therefore, we established an assay to verify the quality of

294 human mRNA in SSLs. The reverse primer was designed near the 3' end and was used with the

295 forward primers to amplify $363 \mathrm{bp}$ and $57 \mathrm{bp}$ long human $A C T B$ mRNA. After reverse

296 transcription using oligo-dT primers, $A C T B$ levels were quantified by performing qPCR using

297 primers generating $363 \mathrm{bp}$ and $57 \mathrm{bp}$ human $A C T B$ mRNA. In the case of $A C T B$ mRNA of

298 longer than $363 \mathrm{bp}$, both 363 and $57 \mathrm{bp}$ fragments were amplified, whereas in case of $A C T B$

299 mRNA of 57-363 bp, only the 57 bp fragment was amplified (Fig. 1b). This assay was validated

300 using RNA subjected to artificial and gradual degradation using RNase. The gradually degraded

301 RNA was analyzed to calculate the percentage of fragments containing $>200$ nucleotides

302 (DV200 value) (Fig. 1c, d). The DV200 values of gradually degraded RNAs negatively 
304 human mRNA degradation in SSLs collected from six males was calculated using a standard

\section{Sebum lipids inhibit RNase activity}

308 Consistent with the results of a study reporting RNase 7 expression in human skin (19), we

309 confirmed that RNase 7 was expressed in the sebaceous glands and epidermis and also detected

310 in SSLs (Fig. 2a, b). Because there is high abundance of RNase on the surface of human skin, it

311 was surprising to detect human mRNA in SSLs. This finding led us to hypothesize that sebum

312 lipids inhibit RNase activity. Based on these results, we evaluated the influence of sebum lipids

313 on recombinant RNase 7 activity. Intact cellular RNA and RNase 7 were incubated with or

314 without sebum lipids from four subjects at $37^{\circ} \mathrm{C}$ for $30 \mathrm{~min}$. Interestingly, the $28 \mathrm{~S}$ and $18 \mathrm{~S}$

315 ribosomal RNAs were completely degraded after incubation with RNase 7 in the absence of

316 sebum lipids; on the other hand, ribosomal RNAs were stable in the presence of lipids (Fig. 2c).

317 Next, we aimed to identify the key lipids that inhibit RNase 7 activity. The sebum lipids were

318 separated into fractions A-D on a TLC plate (Fig. 2d), and the lipids were recovered from each

319 fraction and subjected to the RNase 7 inhibition assay. We observed that while fractions $\mathrm{A}, \mathrm{B}$,

321 Triglycerides and esters in the sebum are hydrolyzed by the skin microbiome to generate free 
322 fatty acids (FFAs). The FFAs in human sebum are predominantly composed of 16 carbon atoms

323 (palmitic acid, 16:0; sapienic acid, 16:1 $\Delta 6$; and palmitoleic acid, C16:1 $\Delta 9$ ) (14). Therefore, we

324 evaluated the inhibitory effects of free palmitoleic acids and various lipids with palmitoleic acid

325 as their main fatty acid, on RNase activity. The free palmitoleic acids and other lipids was

326 dissolved in the reaction buffer at $1 \mathrm{mg} / \mathrm{mL}$ or $100 \mathrm{mg} / \mathrm{mL}$ concentration, except cholesterol and

327 wax esters, and cholesterol, which could not be dissolved at $100 \mathrm{mg} / \mathrm{mL}$. Our results showed

328 that FFAs strongly suppressed RNase activity at $1 \mathrm{mg} / \mathrm{mL}$ compared to other lipids (Fig. 2f).

329 Moreover, FFAs of different chain lengths (myristoleic acid (C14:1) and oleic acid (C18:1)) also

330 suppressed RNase 7 activity (Fig. 2g).

\section{Global expression analysis of SSL-RNAs}

For specific and comprehensive quantification of human mRNAs in SSLs, we performed the

AmpliSeq transcriptome analysis that can perform multiplexed amplification of cDNA

amplicons for more than 20,000 genes. Moreover, this method can analyze even small amounts

of RNAs as well as degraded RNAs $(20,21)$. Although we attempted to prepare sequence

libraries from SSL-RNAs based on the default protocol, our success rate was low. Since the data 
340 the volume of reagents, standardized the conditions for reverse transcription and target

341 amplification, and added a purification step after the target amplification to remove the primer

342 dimers (Supplementary Method 1). With the new protocol, the success rate of the library

343 preparation improved significantly and the AmpliSeq library was prepared with samples

344 obtained from healthy subjects $(91 \%, 29 / 32)$ and patients with $\mathrm{AD}(100 \%, 30 / 30)$. To analyze

345 the experimental bias of our protocol, we verified the correlation between the expression results

346 of AmpliSeq and qPCR of thymus and activation-regulated chemokine (TARC/CCL17) and

347 corneodesmosin $(C D S N)$, and observed a high correlation $(C C L 17, \mathrm{R}=0.81, C D S N, \mathrm{R}=0.87)$

348 (Fig. 3a). Furthermore, the correlation coefficients for the technical replicate of the reverse

349 transcription (0.94 and 0.90) confirmed that our protocol had low experimental bias (Fig. 3b).

The SSL-RNA expression profile predominantly reflects mRNA expression in sebaceous

glands, epidermis, and hair follicles

The regions of the sebaceous glands, epidermis, sweat glands, hair follicles, and dermis

were isolated from the human skin sections using LMD followed by AmpliSeq transcriptome

analysis (Supplementary Fig. 1a). Each region generated distinct clusters when 
expressed in each region (Supplementary Fig. 1c) (23-38). Genes encoding ELOVL fatty acid in other regions (Supplementary Fig. 1c). Other regions isolated by LMD also expressed region-

(normalized counts +1$)>10$, and with more than 1.5-fold differential expression compared to

listed epidermal genes with more than 1.5 -fold differential expression than in sebaceous glands,

RNA profile of 29 healthy subjects was analyzed using the genes expressed characteristically at 
376 SSL-RNAs (Fig. 4). The genes encoding keratin 25, 27, and 71 (KRT25, KRT27, and KRT71,

377 respectively) that are expressed in the inner root sheath of hair follicles $(33,34)$ were highly

378 expressed in SSL-RNAs (Fig. 4). These results suggest that SSL-RNAs provide significant

379 information regarding the granular layer of the epidermis and the inner root sheath of hair

380 follicles.

381

Comparison of SSL-RNAs expression between healthy subjects and patients with AD

We analyzed SSL-RNAs from 29 healthy subjects and 30 patients with AD. The major

output of the AmpliSeq data obtained with our modified method was as follows: i) the average

number of reads was $11,456,318$ in healthy subjects and $11,137,677$ in patients with $A D$; ii) the

expression in AD. Consistent with previous reports (39-42), our results in SSL-RNAs analysis 
394 analysis in skin biopsy samples, genes related to the terminal differentiation of keratinocytes

395 were significantly downregulated and those related to immune-mediated inflammation were

396 upregulated in patients with AD compared with their expression levels in healthy subjects (39).

397 Based on this report, we selected 12 genes related to terminal differentiation and 22 genes

398 related to immune-mediated inflammation that were detected in SSL-RNAs and compared their

399 expression patterns. Our results showed that the expression patterns of these genes in SSL-

400 RNAs of patients with AD and healthy subjects were largely consistent with the previous report

$401 \quad$ (Fig. 5b).

402 Moreover, the analysis of the dimensionality reduction using t-distributed stochastic

403 neighbor embedding (t-SNE) and variance stabilizing transformation (VST) values in all genes

404 showed that the healthy subjects and patients with AD could be distinctly classified into two

405 groups (Fig. 6a). To identify the differential biological functions between healthy subjects and

406 patients with $\mathrm{AD}$, we extracted 918 upregulated and 1,033 downregulated genes in patients with

407 AD (Fig. 6b). In the 833 upregulated genes, GO terms of "mRNA splicing" and "stimulatory C-

408 type lectin receptor signaling pathway" were significantly enriched, while GO term "detection

409 of chemical stimulus involved in sensory perception of smell and keratinocyte differentiation"

410 was enriched in the 951 downregulated genes (Fig. 6c).

411 The sebum secretion is reduced in patients with AD than in healthy individuals (43); 
412 however, the molecular mechanism underlying this reduction remains unknown. GO analysis

413 was performed on 46 genes highly expressed in the sebaceous glands selected from the results

414 of the LMD experiment (Fig. 4), which resulted in the enrichment of genes involved in lipid

415 metabolism (Fig. 7a). The expression of 25 genes involved in lipid metabolism (GO:0006629)

416 was downregulated in patients with AD compared to healthy individuals (Fig. 7b). Furthermore,

417 genes encoding peroxisome proliferator-activated receptor alpha (PPARA), peroxisome

418 proliferator-activated receptor gamma (PPARG), MYC proto-oncogene, bHLH transcription

419 factor $(M Y C)$, transforming growth factor beta 1 (TGFB1), tumor protein $\mathrm{p} 53$ (TP53), and

$420 \mathrm{PR} / \mathrm{SET}$ domain 1 (BLIMPl) regulate sebocyte differentiation and sebum production in vivo and

421 ex vivo (44-47). Among these gene, the expression of TGFB1 was significantly upregulated in

422 patients with AD than in healthy subjects (Fig. 7c). 

oil blotting film.

AmpliSeq transcriptome sequencing. We analyzed the lipid components responsible for 
components, as well as the molecular interactions between them, is necessary to understand the

443 precise stabilization mechanism of mRNA on the skin.

444 We established the method for comprehensive analysis of SSL-RNAs. Recent advances in

445 sequence technology have made it possible to analyze even degraded mRNA. To prepare RNA-

446 seq libraries, a minimum DV200 value of $30 \%$ is generally recommended. Using our modified

447 method, the DV200 of human mRNAs in SSLs was approximately $56.5 \%$, making it suitable

448 for transcriptome analysis. However, when the sequence libraries were prepared according to

449 the standard protocol of Ion AmpliSeq Transcriptome Human Gene Expression Kit, the success

450 rate was very low. Use of the optimized protocol (by standardizing conditions of reverse

transcription and target amplification, and adding a purification step after target amplification)

led to significant improvement in library production efficiency, and the transcriptome

low and the results showed high correlation with qPCR results. Thus, our SSL-RNA analysis

method using the improved AmpliSeq protocol enables profiling of the mRNA expression in

SSLs in a reliable manner. 
analysis of different regions of the skin obtained by LMD showed that the mRNAs derived from

461 the sebaceous glands were highly expressed in SSL-RNAs. However, SSL-RNAs were rich not

462 only in mRNAs derived from sebaceous glands but also in those derived from the epidermis and

463 hair follicles, the tissues in close contact with the sebum. In contrast, the mRNAs characteristic

464 of sweat glands and dermis, which are not in close contact with sebum, were absent in SSLs.

465 The mechanism underlying transfer of epidermal mRNAs into SSLs remain unclear, and

466 there could be several possible mechanisms. The mRNAs characteristic of the granular layer of

467 the epidermis (transcribed from FLG, FLG2 and ASPRV1) were highly expressed in SSL-RNAs.

468 In addition, stratum corneum is reported to contain detectable amounts of mRNA (13-15). We

469 speculate that the epidermal mRNAs transferred to the surface of the stratum corneum due to

470 keratinization are mixed with the sebum on the skin surface, leading to their presence in SSLs.

471 The SSLs also contained hair follicle-derived RNAs, which may be related to the anatomical

472 features of the hair follicle. SSL-RNAs were rich in mRNAs of KRT25, KRT27, and KRT71, the

473 marker genes for the inner root sheath $(33,34)$. The inner root sheath detaches from the hair

474 shaft and degrades during hair growth, and the process occurs at the orifice of the sebaceous

475 duct (51). These observations indicate that the epithelial cells of the inner root sheath may get

476 mixed with sebum at the orifice and skin surface, and as a result, information pertaining to the 
478 released from various cells contain several biomolecules including mRNAs $(52,53)$, it is

479 possible that extracellular vesicles-derived mRNAs are also included in the SSL-RNAs.

480 Collectively, our results indicate that SSL-RNAs predominantly contains mRNAs derived from

481 the sebaceous glands, epidermis, and hair follicles, and are therefore, a useful resource for

482 analyzing the biological information related to the relevant regions of the skin.

483 Finally, we verified the applicability of this method by performing a comparative analysis of

484 the SSL-RNAs profiles of healthy subjects and patients with AD. We observed that the

transcriptome profile was markedly different between healthy subjects and patients with $\mathrm{AD}$,

with differential expression of immune-mediated inflammation and terminal differentiation-

related genes, as shown in a previous skin biopsy report (39). Moreover, the GO term "detection

of chemical stimulus involved in sensory perception of smell" identified in our study was

consistent with a previous report based on patients with $\mathrm{AD}$ (13) indicating that the analysis of

SSL-RNAs successfully captured the characteristics of AD. Atrophy of the sebaceous glands

and reduction in sebum secretion have been reported in patients with AD (54). However, little is

known about the underlying mechanism, including the gene expression profile of sebaceous

glands, in patients with $\mathrm{AD}$ due to difficulty in obtaining facial skin tissue samples containing 
496 Moreover, the expression of $T G F B 1$, which suppresses sebocyte differentiation and lipid

497 accumulation (47), was significantly increased in patients with $\mathrm{AD}$, suggesting that the

498 suppression of lipid synthesis via TGFB1 may be one of the mechanisms responsible for

499 dysregulated sebum synthesis in these patients. Thus, the transcriptome analysis of SSL-RNAs

500 can evaluate the molecular profile of $\mathrm{AD}$ in a non-invasive manner, and is a promising method

501 for comprehensive understanding of AD pathology.

502 In summary, we established a non-invasive method for SSL-RNA analysis that utilizes SSL

503 samples collected by simply wiping the skin surface for less than a minute. This non-invasive

504 method has potential application in unraveling the molecular profile of skin diseases, such as

$505 \mathrm{AD}$, which will be helpful for clinical management of these diseases in future. Understanding

506 the status and course of AD at the molecular level is essential not only to assess the

507 pathophysiology of AD but also to design its effective therapeutic treatments. The clinical

508 phenotypes of AD are extremely complex, warranting the need for identifying biomarkers that

509 can classify invisible endophenotypes (55). Further, since the skin is called "the disease-sensor

510 organ" (56) and is believed to reflect the conditions inside the body, the SSL-RNA analysis may

511 have wide applicability to understand various pathologies of human body. 


\section{Data availability}

514 The datasets generated and analyzed in the current study are available from the

515 corresponding author on reasonable request.

\section{$517 \quad$ References}

518 1) Droujinine, I. A. \& Perrimon, N. Interorgan communication pathways in physiology: focus on Drosophila. Annu. Rev. Genet. 50, 539-570 (2016).

2) Heikenfeld, J. et al. Accessing analytes in biofluids for peripheral biochemical monitoring. Nat. Biotechnol. 37, 407-419 (2019).

3) Yokoi, A. et al. Integrated extracellular microRNA profiling for ovarian cancer screening. Nat. Commun. 9, 4319 (2018).

4) Sudo, K. et al. Development and validation of an esophageal squamous cell carcinoma detection model by large-scale microRNA profiling. JAMA Netw. Open 2, e194573 (2019).

5) Yasui, T. et al. Unveiling massive numbers of cancer-related urinary-microRNA candidates via nanowires. Sci. Adv. 3, e1701133 (2017).

6) Paliwal, S., Hwang, B. H., Tsai, K. Y. \& Mitragotri, S. Diagnostic opportunities based on skin biomarkers. Eur. J. Pharm. Sci. 50, 546-556 (2013). 
532 8) Gao, W. et al. Fully integrated wearable sensor arrays for multiplexed in situ perspiration analysis. Nature 529, 509-514 (2016).

9) Moyer, J., Wilson, D., Finkelshtein, I., Wong, B. \& Potts, R. Correlation between sweat glucose and blood glucose in subjects with diabetes. Diabetes Technol. Ther. 14, 398-402 (2012).

10) Park, J. H. P. et al. An antimicrobial protein, lactoferrin exists in the sweat: proteomic analysis of sweat. Exp. Dermatol. 20, 369-371 (2011).

11) Bengtsson, C. F. et al. DNA from keratinous tissue. Part I: hair and nail. Ann. Anat. 194, $17-$ 25 (2012).

12) Wong, R. et al. Use of RT-PCR and DNA microarrays to characterize RNA recovered by non-invasive tape harvesting of normal and inflamed skin. J. Invest. Dermatol. 123, 159167 (2004).

13) Dyjack, N. et al. Minimally invasive skin tape strip RNA sequencing identifies novel characteristics of the type 2-high atopic dermatitis disease endotype. J. Allergy Clin. Immunol. 141, 1298-1309 (2018). psoriasis. J. Allergy Clin. Immunol. 147, 199-212 (2021). 
549 15) Pavel, A. B. et al. Tape strips from early-onset pediatric atopic dermatitis highlight disease abnormalities in nonlesional skin. Allergy. 76, 314-325 (2021).

16) Leung, D. Y. M. et al. The nonlesional skin surface distinguishes atopic dermatitis with food allergy as a unique endotype. Sci. Transl. Med. 11, eaav2685 (2019).

17) Peppelman, M., van den Eijnde, W. A. J., Jaspers, E. J., Gerritsen, M-J. P. \& van Erp, P. E. J. model to study skin damage. Skin Res. Technol. 21, 474-484 (2015).

18) Schneider, M. R. \& Paus, R. Sebocytes, multifaceted epithelial cells: lipid production and holocrine secretion. Int. J. Biochem. Cell Biol. 42, 181-185 (2010). faecium. PLoS One 4, e6424 (2009).

20) Li, W. et al. Comprehensive evaluation of AmpliSeq transcriptome, a novel targeted whole Genomics 16, 1069 (2015). for RNA-seq analysis of the human allograft kidney biopsy tissue. BMC Med. Genomics 12, 86 (2019). 
paraffin-embedded diagnostic prostate tumor specimens. Lab. Invest. 98, 537-550 (2018).

23) Westerberg, R. et al. Role for ELOVL3 and fatty acid chain length in development of hair and skin function. J. Biol. Chem. 279, 5621-5629 (2004).

24) Kitago, M. et al. Localization of the candidate genes ELOVL5 and SCD1 for 'male effect' pheromone synthesis in goats (Capra hircus). J. Reprod. Dev. 53, 1329-1333 (2007).

25) Dahlhoff, M. et al. PLIN2, the major perilipin regulated during sebocyte differentiation, controls sebaceous lipid accumulation in vitro and sebaceous gland size in vivo. Biochim. Biophys. Acta 1830, 4642-4649 (2013).

26) Joost, S. et al. Single-cell transcriptomics reveals that differentiation and spatial signatures shape epidermal and hair follicle heterogeneity. Cell Syst 3, 221-237.e9 (2016).

27) Zhang, Y. et al. Activation of beta-catenin signaling programs embryonic epidermis to hair follicle fate. Development 135, 2161-2172 (2008).

28) Makino, T., Mizawa, M., Yamakoshi, T., Takaishi, M. \& Shimizu, T. Expression of filaggrin-2 protein in the epidermis of human skin diseases: a comparative analysis with filaggrin. Biochem. Biophys. Res. Commun. 449, 100-106 (2014).

29) Donovan, M. et al. Filaggrin and filaggrin 2 processing are linked together through skin aspartic acid protease activation. PLoS One 15, e0232679 (2020). 
epithelial cells. Int. J. Mol. Med. 34, 997-1003 (2014). produced by eccrine sweat glands and is not induced in epidermal cells under inflammatory skin conditions. Br. J. Dermatol. 151, 534-539 (2004).

32) Miura, K. et al. Homeobox transcriptional factor engrailed homeobox 1 is expressed (2018).

33) Langbein, L. et al. K25 (K25irs1), K26 (K25irs2), K27 (K25irs3), and K28 (K25irs4) represent the type I inner root sheath keratins of the human hair follicle. J. Invest. Dermatol. 126, 2377-2386 (2006).

34) Langbein, L., Yoshida, H., Praetzel-Wunder, S., Parry, D. A. \& Schweizer, J. The keratins of the human beard hair medulla: the riddle in the middle. J. Invest. Dermatol. 130, 55-73 (2010).

35) Kutz, W. E. et al. ADAMTS10 protein interacts with fibrillin-1 and promotes its deposition in extracellular matrix of cultured fibroblasts. J. Biol. Chem. 286, 17156-17167 (2011). 
603 37) Warrick, E. et al. Morphological and molecular characterization of actinic lentigos reveals

604 alterations of the dermal extracellular matrix. Br. J. Dermatol. 177, 1619-1632 (2017).

605 38) Moustou, A. E. et al. Expression of lymphatic markers and lymphatic growth factors in

606 psoriasis before and after anti-TNF treatment. An. Bras. Dermatol. 89, 891-897 (2014).

607 39) Suárez-Fariñas, M. et al. Nonlesional atopic dermatitis skin is characterized by broad

608 terminal differentiation defects and variable immune abnormalities. J. Allergy Clin.

609 Immunol. 127, 954-64.e1-4 (2011).

610 40) Nomura, I. et al. Cytokine milieu of atopic dermatitis, as compared to psoriasis, skin prevents induction of innate immune response genes. J. Immunol. 171, 3262-3269 (2003).

612 41) Bianchi, P. et al. Analysis of gene expression in atopic dermatitis using a microabrasive method. J. Invest. Dermatol. 132, 469-472 (2012).

42) Gittler, J. K. et al. Progressive activation of T(H)2/T(H)22 cytokines and selective epidermal proteins characterizes acute and chronic atopic dermatitis. J. Allergy Clin. Immunol. 130, 1344-1354 (2012).

43) Furuichi, M. et al. The usefulness of sebum check film for measuring the secretion of sebum. Arch. Dermatol. Res. 302, 657-660 (2010). production. J. Invest. Dermatol. 126, 2002-2009 (2006). 
621 45) Cottle, D. L. et al. c-MYC-induced sebaceous gland differentiation is controlled by an androgen receptor/p53 axis. Cell Rep. 3, 427-441 (2013).

46) Horsley, V. et al. Blimp1 defines a progenitor population that governs cellular input to the sebaceous gland. Cell 126, 597-609 (2006).

47) McNairn, A. J. et al. TGF $\beta$ signaling regulates lipogenesis in human sebaceous glands cells. BMC Dermatol. 13, 2 (2013).

48) Sorrentino, S. The eight human "canonical" ribonucleases: molecular diversity, catalytic properties, and special biological actions of the enzyme proteins. FEBS Lett. 584, 2194$2200(2010)$.

630

49) Lambers, H., Piessens, S., Bloem, A., Pronk, H. \& Finkel, P. Natural skin surface pH is on (2006).

50) Sheu, H. M., Chao, S. C., Wong, T. W., Yu-Yun Lee, J. \& Tsai, J. C. Human skin surface lipid film: an ultrastructural study and interaction with corneocytes and intercellular lipid lamellae of the stratum corneum. Br. J. Dermatol. 140, 385-391 (1999).

51) Sundberg, J. P. et al. Asebia-2J (Scd1ab2J): A new allele and a model for scarring alopecia. Am. J. Pathol. 156, 2067-2075 (2000). 
640 53) Wu, C.-X. \& Liu, Z.-F. Proteomic profiling of sweat exosome suggests its involvement in skin immunity. J. Invest. Dermatol. 138, 89-97 (2018).

54) Shi, V. Y. et al. Role of sebaceous glands in inflammatory dermatoses. J. Am. Acad. Dermatol. 73, 856-863 (2015).

644 55) Muraro, A. et al. Precision medicine in patients with allergic diseases: airway diseases and atopic dermatitis-PRACTALL document of the European Academy of Allergy and Clinical Immunology and the American Academy of Allergy, Asthma \& Immunology. J. Allergy Clin. Immunol. 137, 1347-1358 (2016).

648 56) Kozawa, S. et al. The body-wide transcriptome landscape of disease models. iScience 2 , 


\section{Author Contributions}

652 T.I. conceived the study. T.I., A.H., Y.T., and T.M. planned the study. T.I., T.K., Y.U., M.Y.,

653 and N.Oy. performed the experiments and analyzed the data. T.M., Y.T., and N.Ot. supervised

654 the research. T.I. and T.M. wrote the manuscript. All authors reviewed the manuscript.

655

656 Competing Interests statement

657 A patent application related to this work has been filed (No. PCT/JP2017/021040,: "Method

658 for preparing nucleic acid sample.” Status: patent granted (DE, FR, GB, KR, JP), patent pending

659 (CN, US). Inventors: T.I. and A.H. Patent applicant: Kao Corporation). All other co-authors

660 declare that they have no competing interests.

661 
a

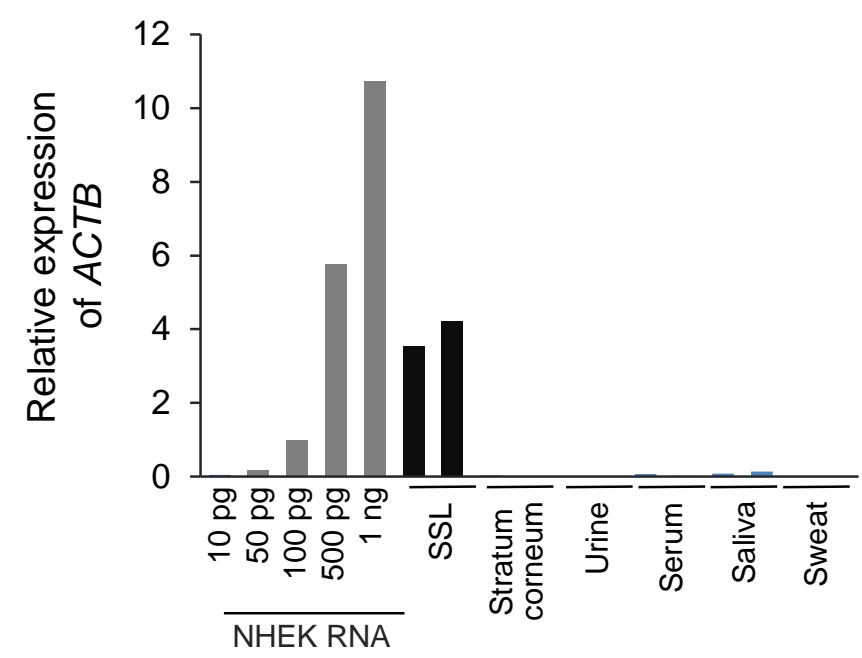

b

Reverse transcription

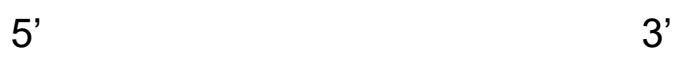

AAAAA

TTTTT

C

(nt)

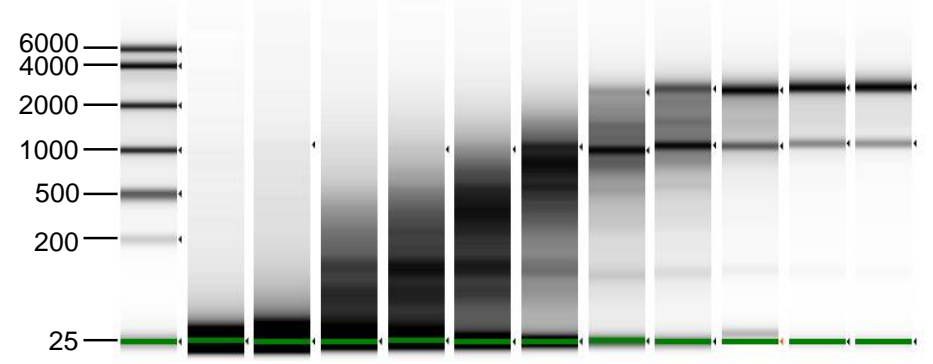

e

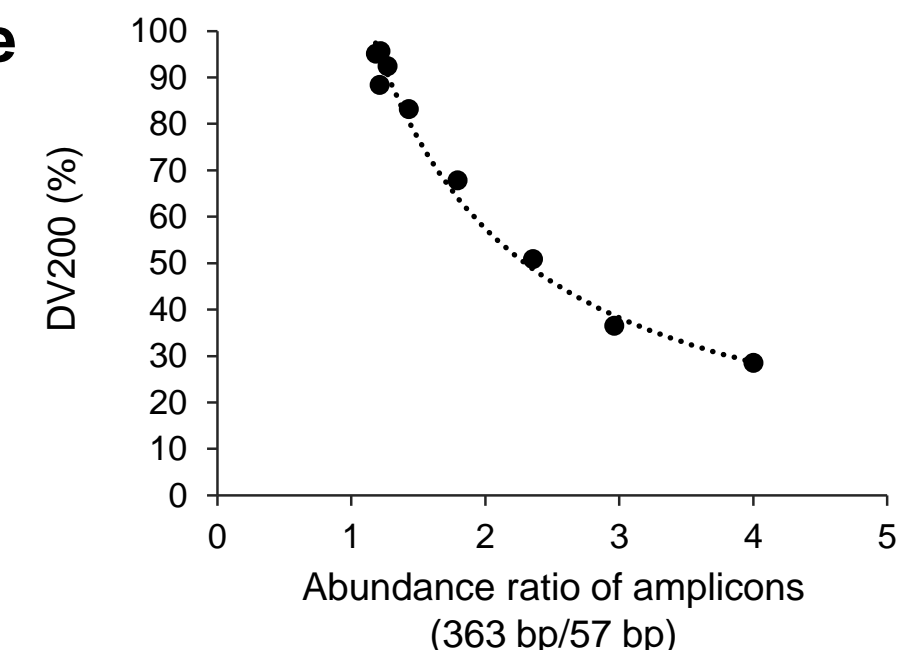

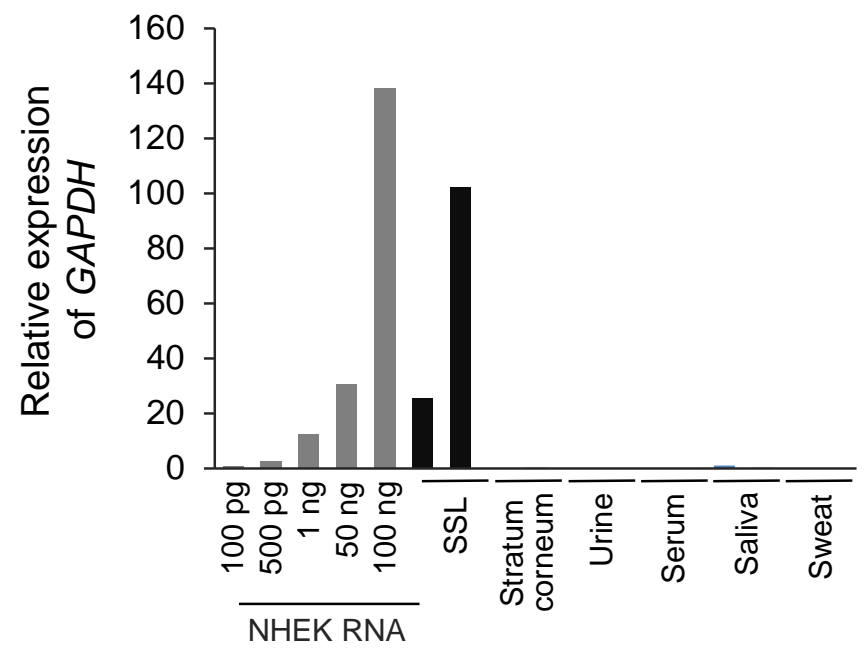

ACTB

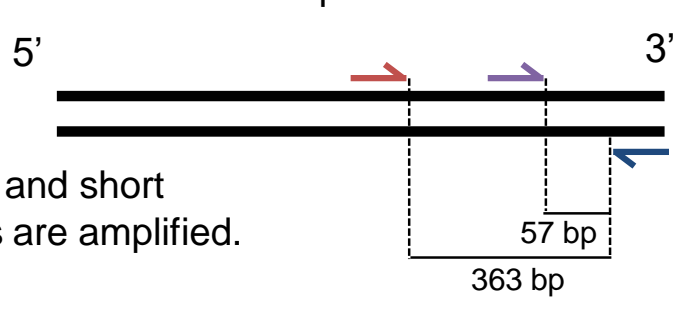

Long fragments

are not amplified.

d

Both long and short

fragments are amplified.
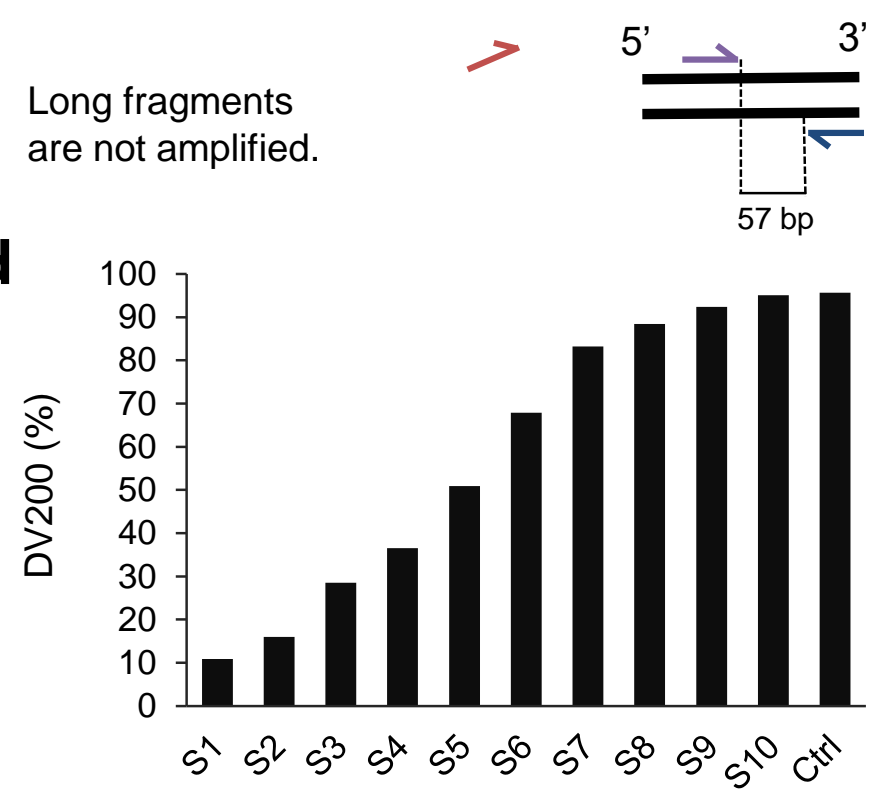

f

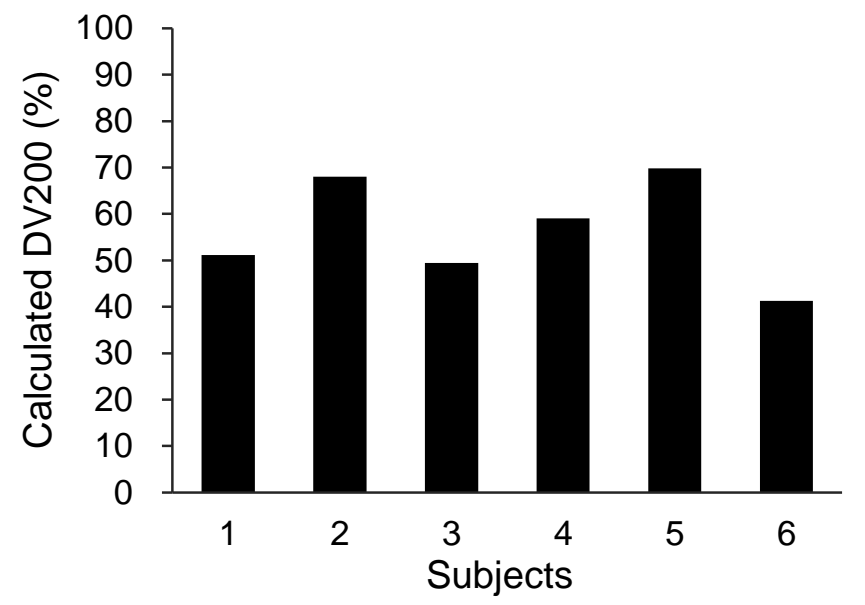


Figure 1. Evaluation of mRNA expression and RNA degradation in skin surface lipids (SSLs)

(a) Expression of $A C T B$ and GAPDH mRNA in SSLs, stratum corneum, urine, serum, saliva, and sweat samples analyzed by qPCR. Gene expression is shown as the relative expression for $100 \mathrm{pg}$ of RNA derived from normal human epidermal keratinocytes (NHEK). NHEK total RNA (10 pg to $100 \mathrm{ng}$ ) is the standard used. (b) Outline for assaying the degradation of human mRNA in SSLs. The extent of mRNA degradation was determined using the long (363 bp)/short (57 bp) amplicon ratio calculated from qPCR results. (c) Preparation of RNA samples with different levels of degradation. A series of standards (S1 to S10) are presented with known level of RNA degradation. (d) DV200 value indicating the percentage of fragments containing > 200 nucleotides, of 1c. (e) The relationship between the DV200 of $1 \mathbf{d}$ and the value of $363 \mathrm{bp} / 57 \mathrm{bp}$ (abundance of $363 \mathrm{bp}$ amplicon/abundance of 57 bp amplicon measured by qPCR).

(f) The indirect assessment of the human mRNA degradation in SSLs, $n=6$. DV200 of each SSL-RNA sample was calculated using the standard curve of $1 \mathbf{e}$. 
a

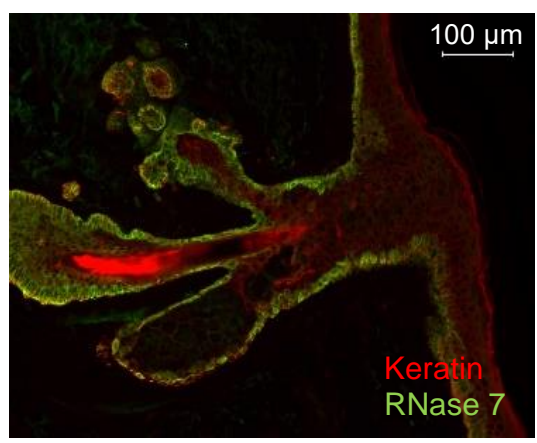

b

(kDa)

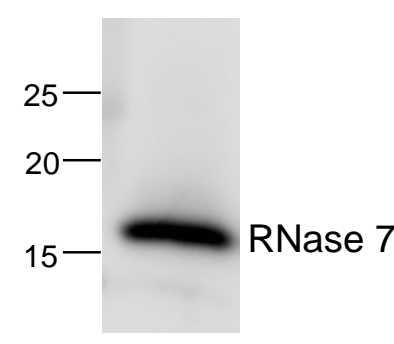

e

d

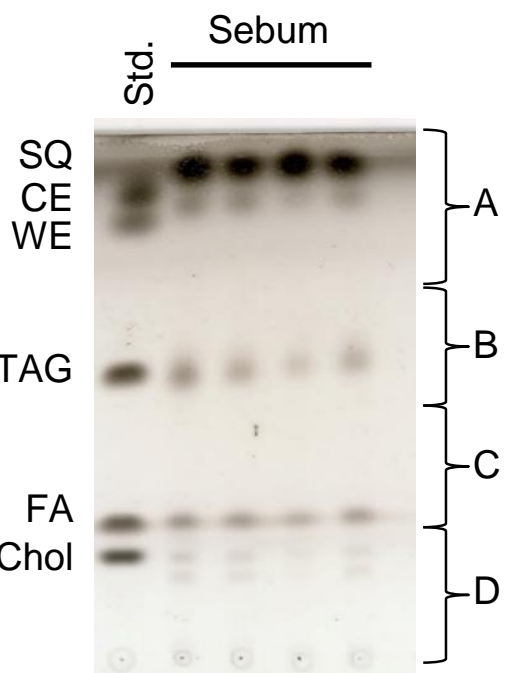

$\begin{array}{llll}123 & 4 & \text { Subjects }\end{array}$

f

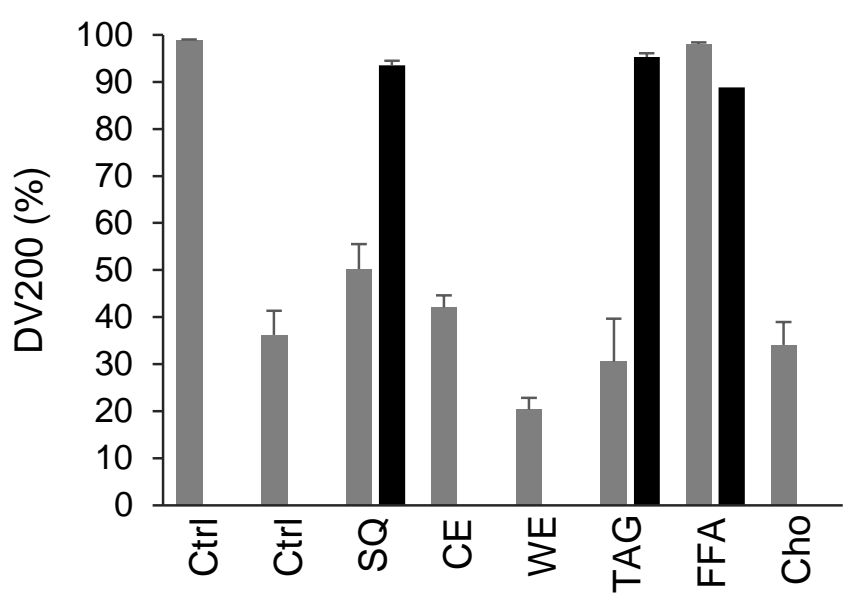

+RNase 7

(nt)

Ctrl

g

\section{C}

(nt)

Ctrl
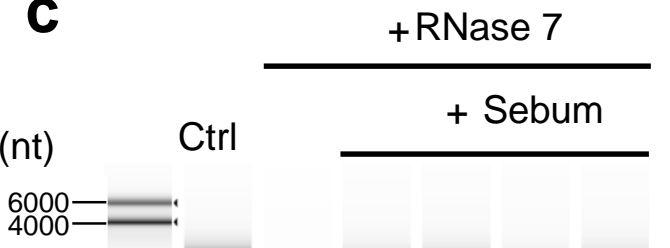

$2000-\square .+28 S$

1000 - . . . $18 \mathrm{~S}$

$500-\longrightarrow$.

200
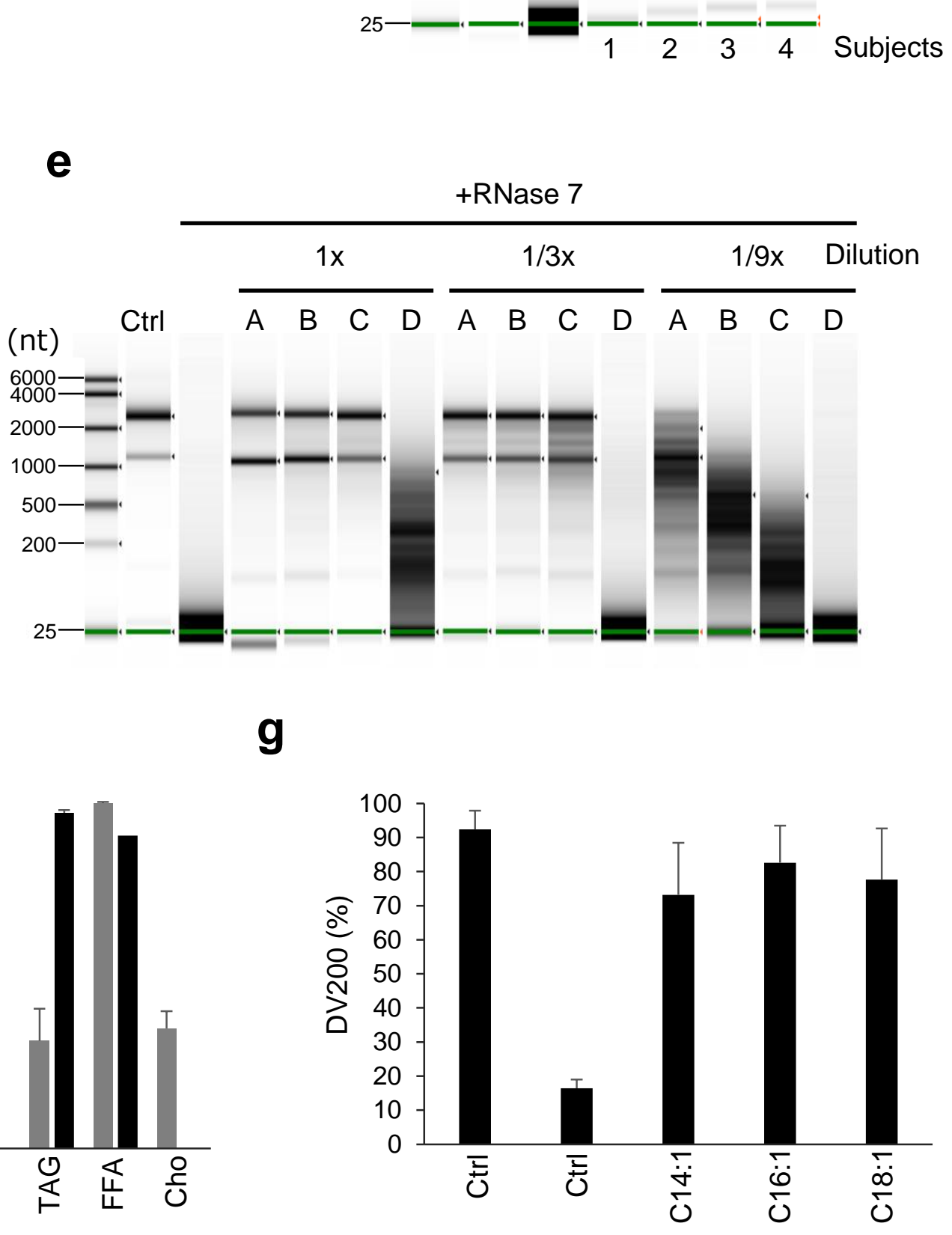

+RNase 7

$: 1 \mathrm{mg} / \mathrm{mL} \quad \square: 100 \mathrm{mg} / \mathrm{mL}$ 
Figure 2. Effect of sebum lipids on RNase activity

(a) The localization of RNase 7 in the human skin. Green, RNase 7; red, keratin/cytokeratin. Bar: 100 $\mu \mathrm{m}$. (b) The detection of RNase 7 in SSLs collected from healthy males by western blotting. (c) Determination of the effect of sebum lipids collected from four healthy males on RNase activity using NHEK total RNA. (d) Fractionation of sebum lipids collected from four healthy males by performing thin-layer chromatography (TLC). The standard lane (Std) includes authentic samples: SQ, squalene; CE, cholesterol ester (cholesteryl palmitate); WE, wax ester (lauryl palmitoleate); TAG, triacylglycerol (glyceryl trioleate); FA, free fatty acid (palmitoleic acid); and Chol, cholesterol. A to D sebum samples were collected, and lipids were extracted from the silica gel for the subsequent assays. (e) Effect of pooled sebum lipids collected from A to D on RNase activity using NHEK RNA. (f) Effect of each lipid on RNase activity using NHEK RNA. The DV200 values are shown as mean $\pm \mathrm{SE}, \mathrm{n}=3$. WE, wax ester (behenyl palmitoleate); TAG, triacylglycerol (glyceryl tripalmitoleate); Ctrl; EtOH. (g) Relationship between RNase activity and chain length of fatty acids. The DV200 values are shown as the mean \pm SE, n = 6. C14:1, myristoleic acid; C16:1, palmitoleic acid; C18:1, oleic acid; Ctrl; EtOH. 


\section{Figure 3}

a

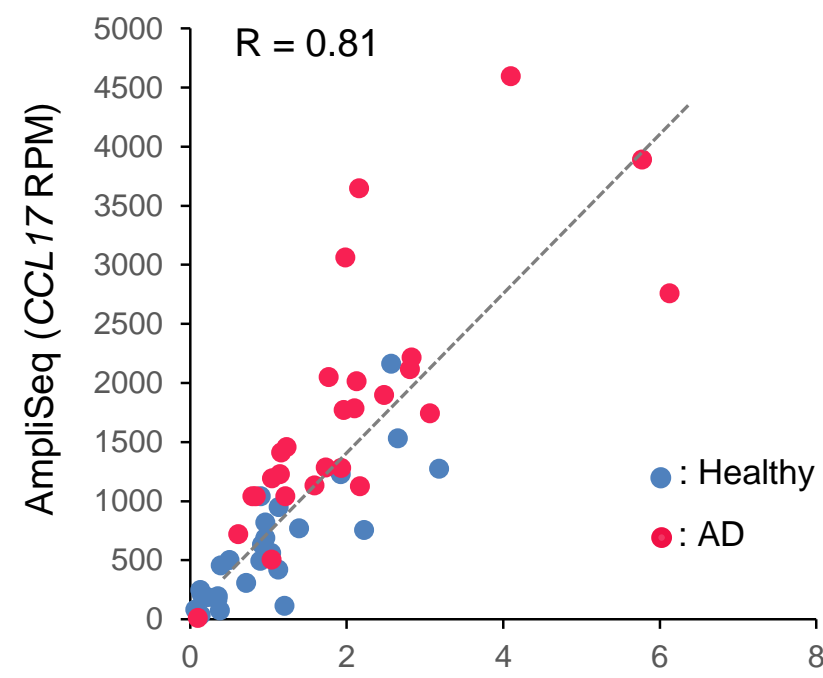

Relative gene expression by qPCR (CCL17/RPLP0)

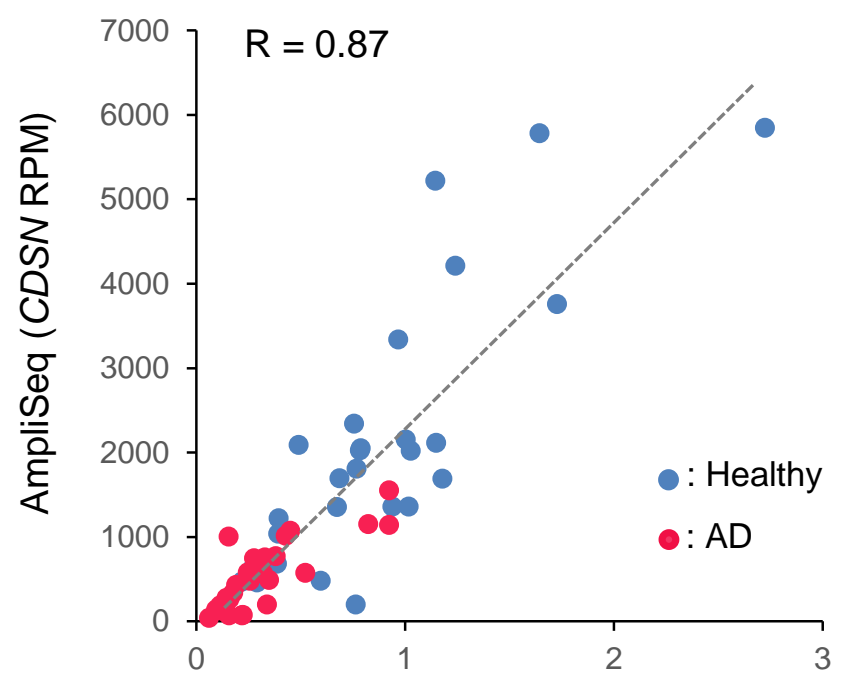

Relative gene expression by qPCR (CDSN/RPLPO)

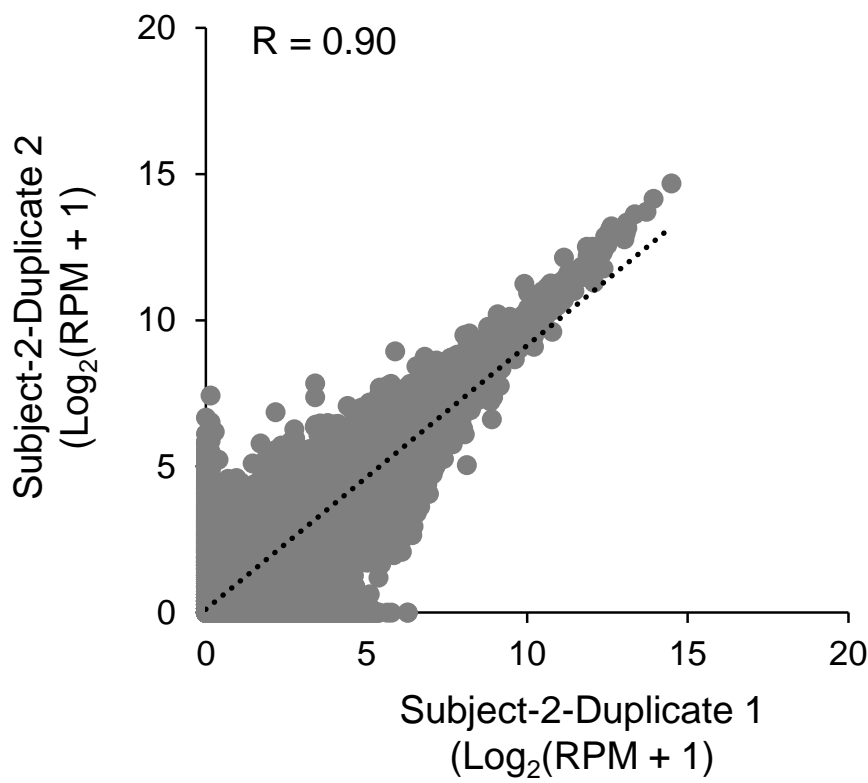

Figure 3. Accuracy of the AmpliSeq data output using the modified protocol

(a) The correlation between AmpliSeq and qPCR results for CCL17 and CDSN expression in healthy subjects $(n=29$, blue $)$ and patients with $\mathrm{AD}(\mathrm{n}=30$, red $)$. (b) The correlation of the transcriptome profile when preparing libraries in duplicate from each SSL-RNA obtained from two healthy male subjects. 


\section{Figure 4}

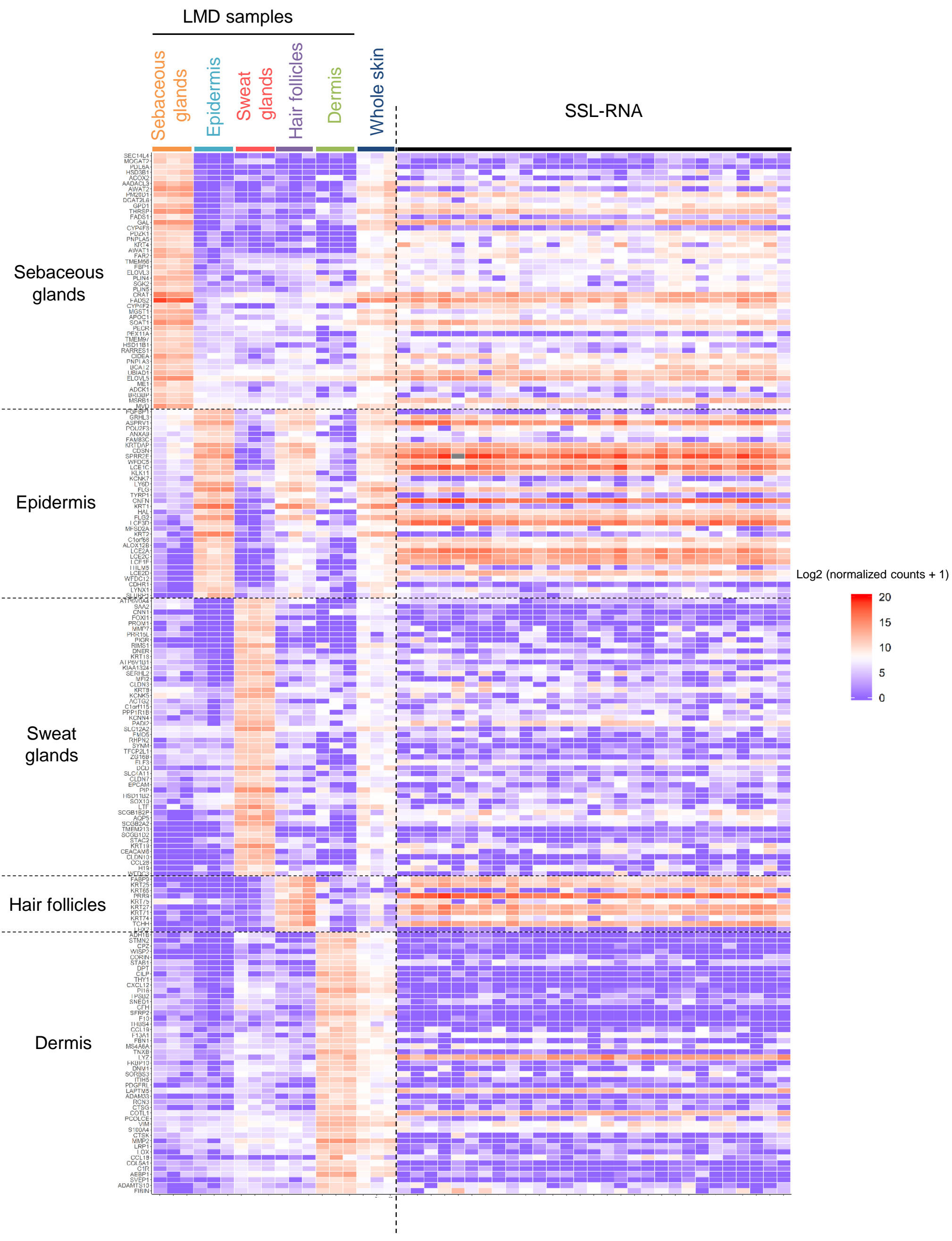


Figure 4. mRNA expression characteristic of different skin regions and its comparison with the expression profile of SSL-RNAs

Heatmap showing the expression profiles for each region (sebaceous glands, epidermis, sweat glands, hair follicles, and dermis) isolated using LMD, whole skin from three healthy male subjects, and SSLRNAs obtained from 29 healthy male subjects. 
Figure 5

a

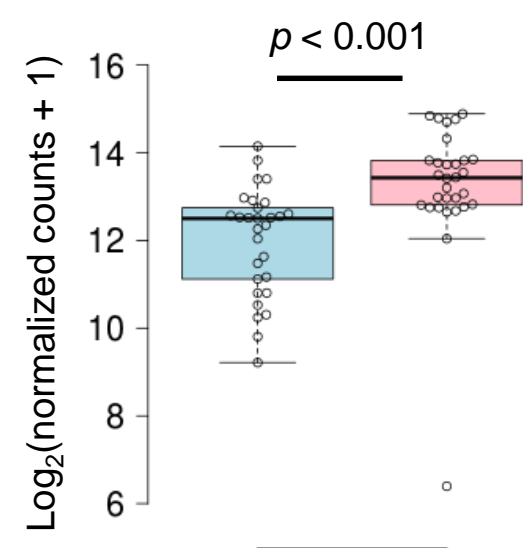

$\mathrm{HL}$

S100A9

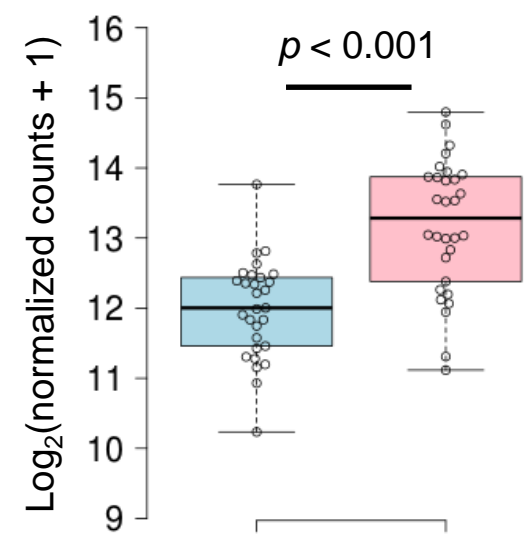

$\mathrm{HL} \quad \mathrm{AD}$

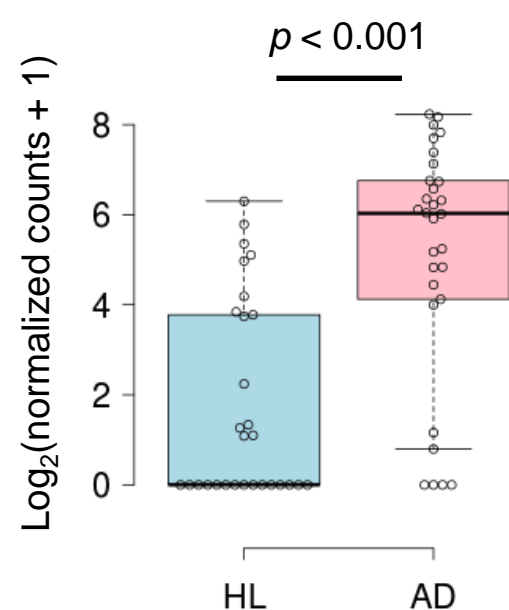

FLG

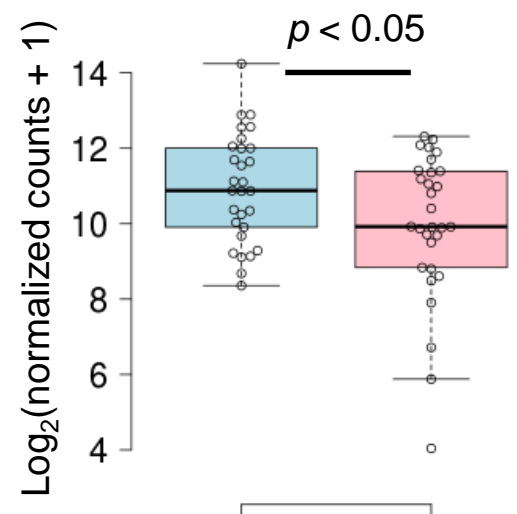

$\mathrm{HL}$
IL13

IL1B

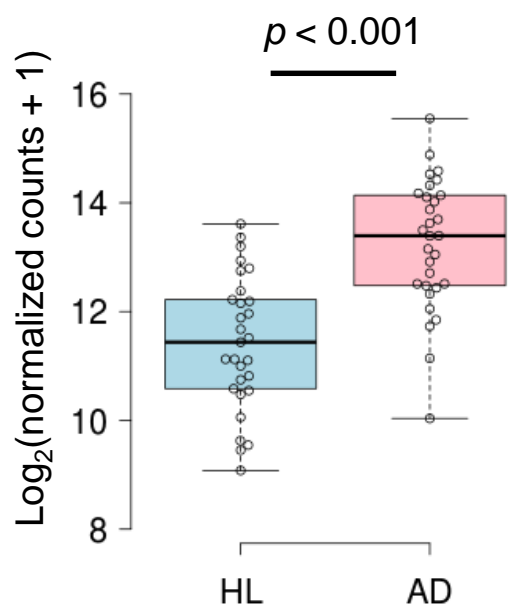

IVL

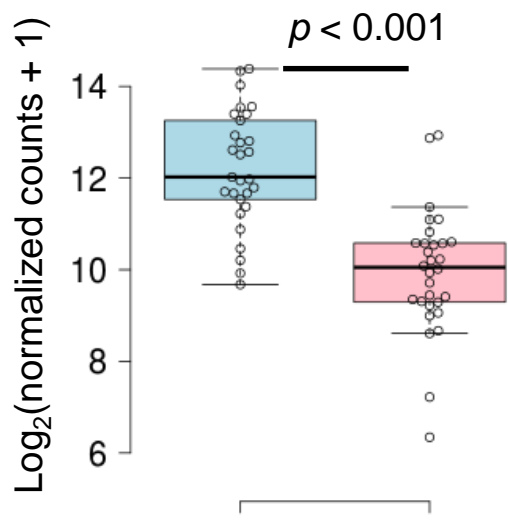

$\mathrm{HL}$

$A D$

b

\section{Terminal differentiation-related genes}

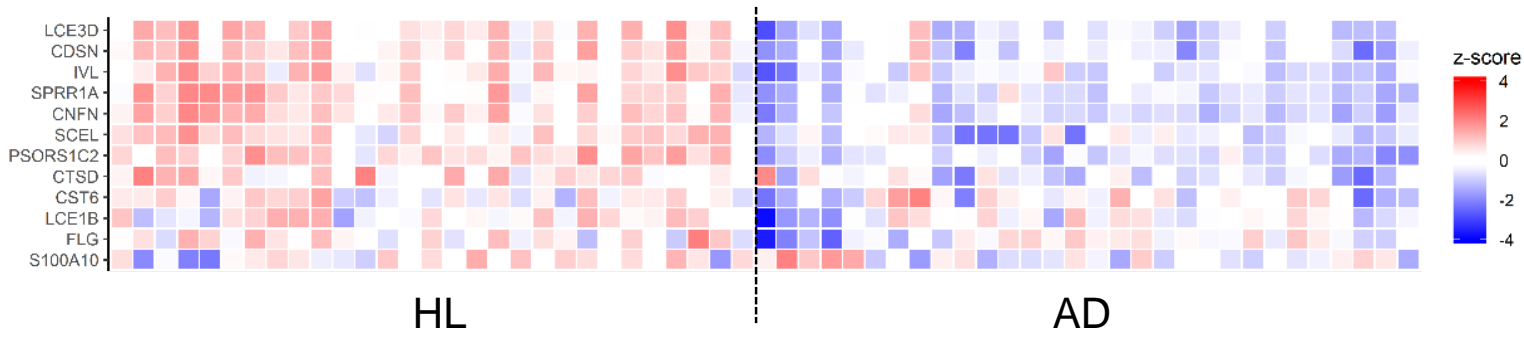

Genes related to immune-mediated inflammation

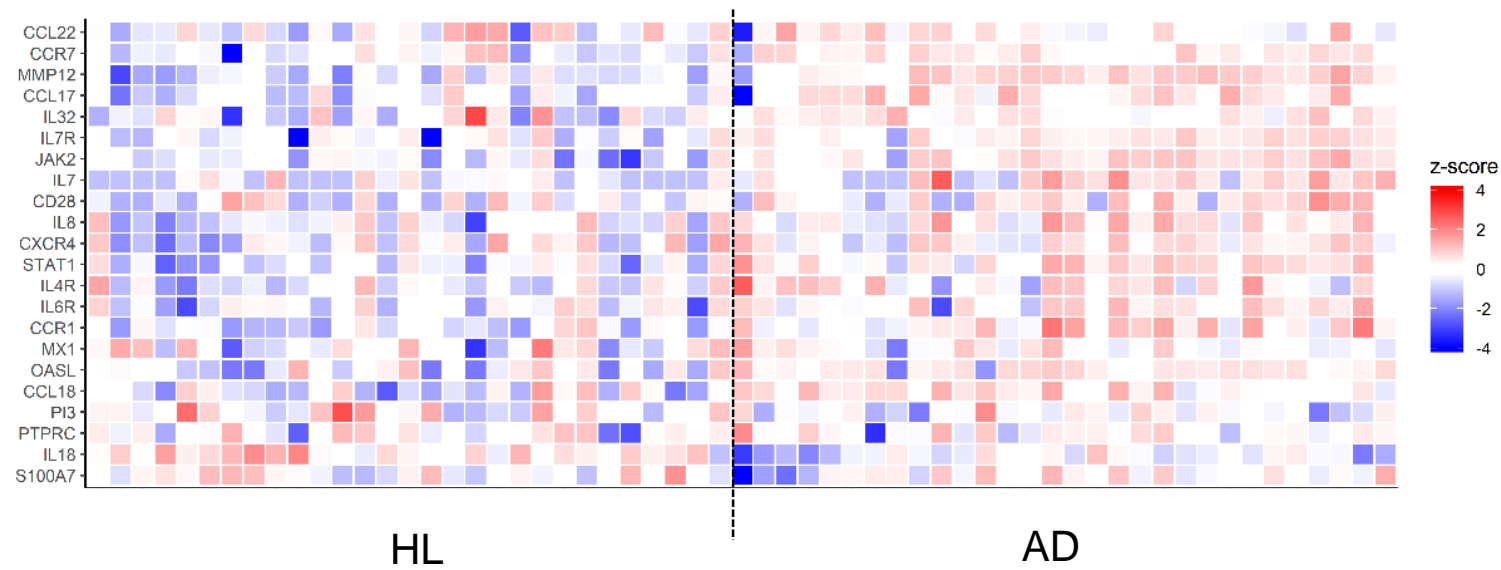


Figure 5. Comparison of AD marker genes in healthy subjects (HL) and patients with AD

(a) The differential expression of CCL17, IL13, IL1B, S100A9, FLG, and IVL in HL and AD. Boxes

represent mean \pm interquartile range $(\mathrm{IQR})$, and whiskers represent 1 st and 3rd quartile $1.5 * \mathrm{IQR}$.

Benjamini-Hochberg adjusted $p$-values are shown from the likelihood ratio test between HL and AD. HL $(n=29), A D(n=30)$. (b) Heatmaps using $z$-transformed $\log _{2}$ (normalized counts +1$)$ in 12 terminal differentiation-related genes (upper) and 22 genes related to immune-mediated inflammation (lower). 


\section{Figure 6}

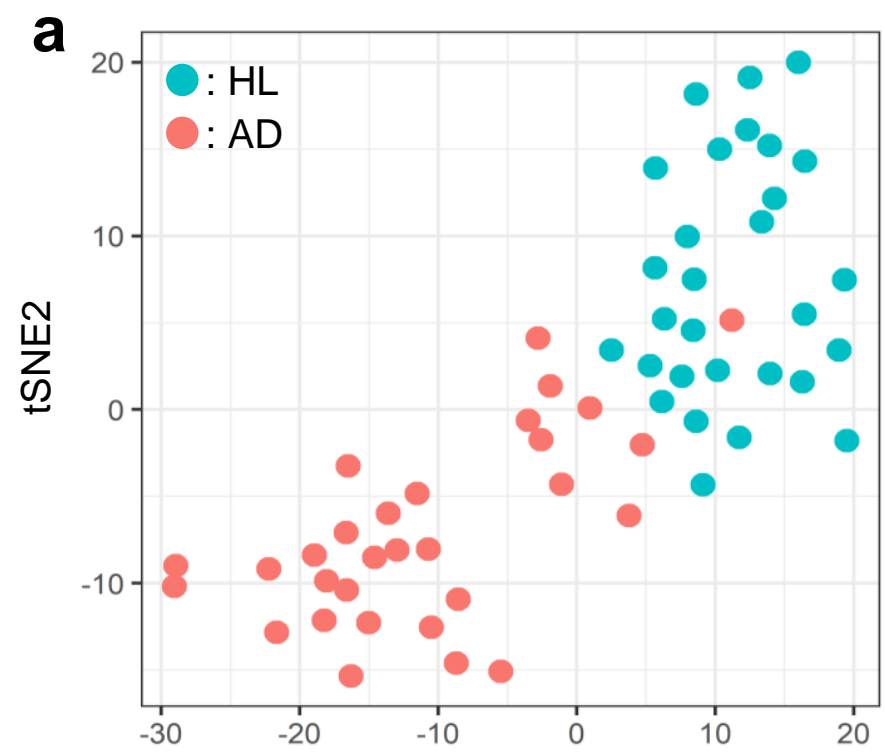

tSNE1

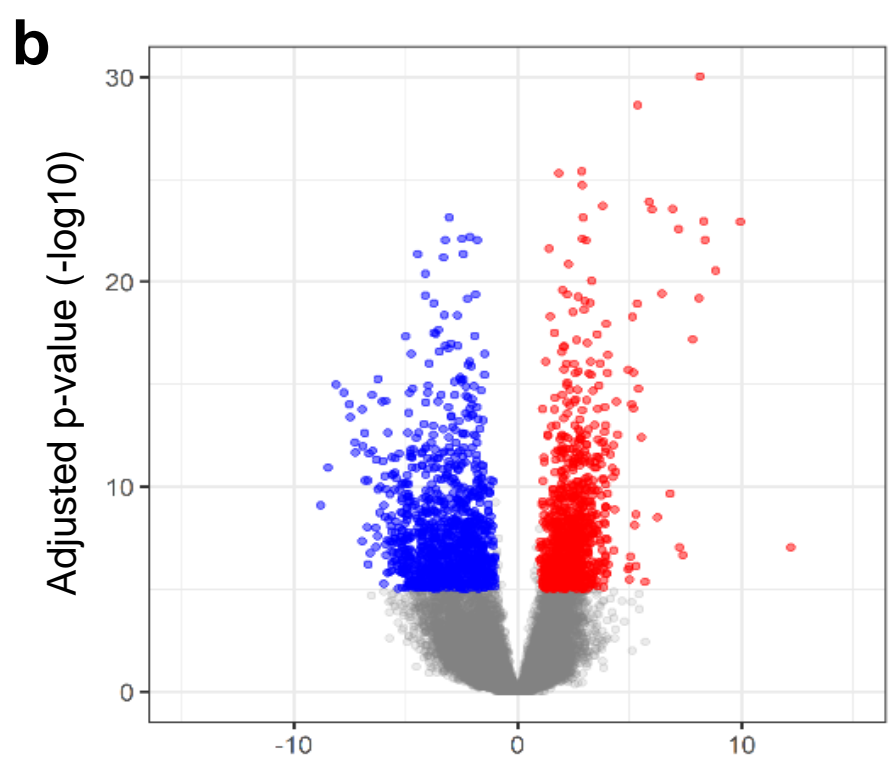

Fold change $(\log 2)$

\section{Upregulated genes in AD}

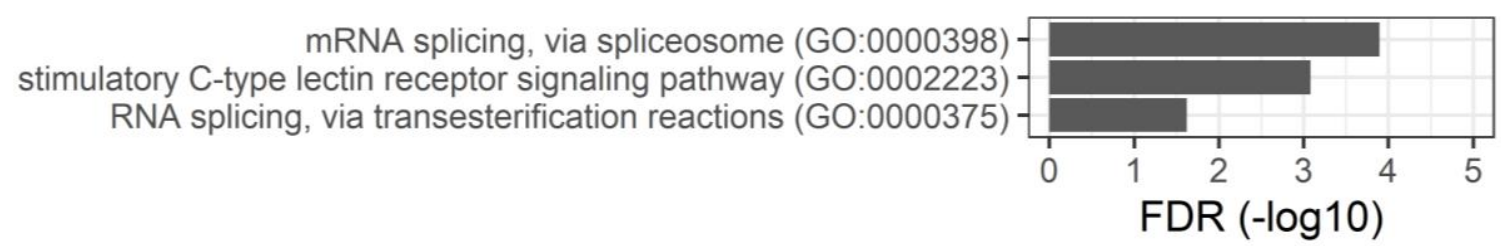

\section{Downregulated genes in AD}

detection of chemical stimulus involved in sensory perception of smell (GO:0050911) G-protein coupled receptor signaling pathway (GO:0007186) detection of chemical stimulus involved in sensory perception (GO:0050907) sensory perception of smell (GO:0007608) peptide cross-linking (GO:0018149) keratinization (GO:0031424) nucleosome assembly (GO:0006334) keratinocyte differentiation (GO:0030216) epidermis development (GO:0008544) innate immune response in mucosa (GO:0002227) antibacterial humoral response (GO:0019731)

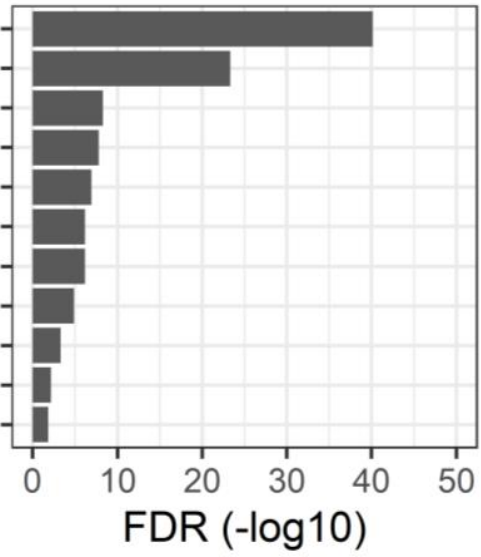

Figure 6. Characterization of SSL-RNAs profiles in healthy subjects (HL) and patients with AD (a) t-SNE analysis using variance stabilizing transformation (VST) values for all genes (green, HL; red, AD). (b) Volcano plot of differentially expressed genes (DEGs) (red, upregulated; blue, downregulated) in patients with AD compared to HL subjects (Benjamini-Hochberg adjusted $p$-value $<10^{-5}$ and fold change > 2.0). (c) Gene ontology analysis of DEGs. The upper panel shows significant biological process (BP) of upregulated DEGs and the lower panel shows BP of downregulated DEGs in patients with AD 


\section{Figure 7}

a

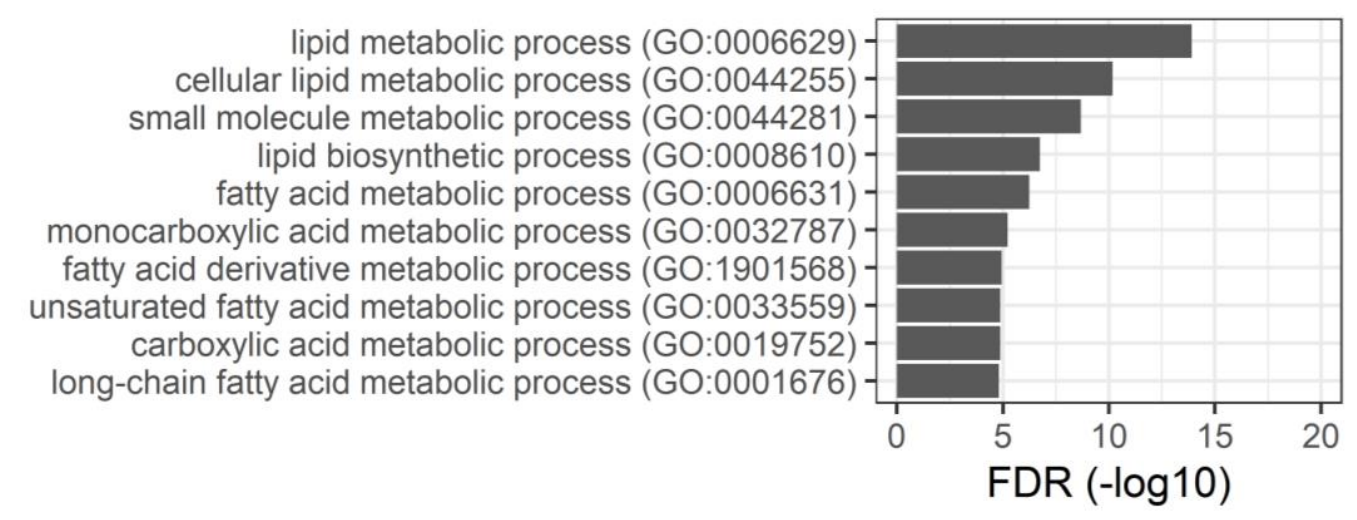

b
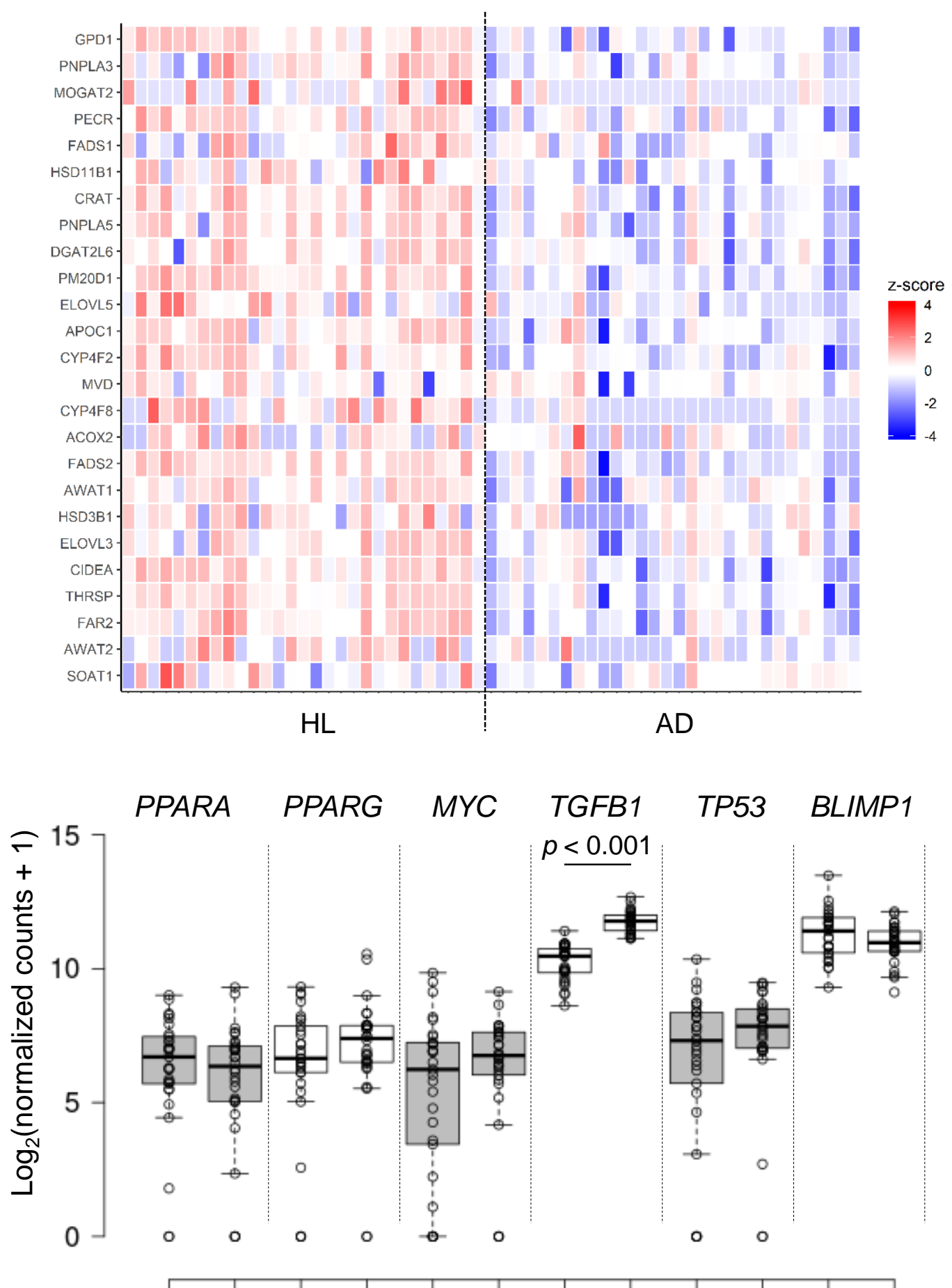

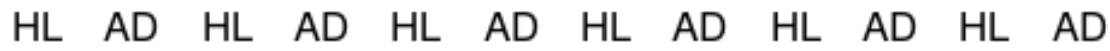


Figure 7. Comparison of SSL-RNAs profile representing highly expressed genes in sebaceous glands in healthy subjects (HL) and patients with AD

(a) Gene ontology analysis of 25 genes highly expressed in sebaceous glands (selected in Fig. 4). (b) Heatmaps using z-transformed $\log _{2}$ (normalized counts +1 ) of 25 genes highly expressed in sebaceous glands. (c) The differential expression of PPARA, PPARG, MYC, TGFB1, TP53, and BLIMP1 in HL and patients with $\mathrm{AD}$. Boxes represent mean \pm interquartile range (IQR), and whiskers represent 1 st and 3 rd quartile $1.5 *$ IQR. Benjamini-Hochberg adjusted $p$-values are shown from the likelihood ratio test between HL and AD. HL ( $n=29)$, patients with AD $(n=30)$. 